\title{
A reduced computational and geometrical framework for inverse problems in haemodynamics
}

\author{
Toni Lassila ${ }^{1}$, Andrea Manzoni $^{3}$, Alfio Quarteroni $^{1,2}$, Gianluigi Rozza $^{3, *, \dagger}$ \\ ${ }^{1}$ CMCS - Modelling and Scientific Computing, MATHICSE - Mathematics Institute of Computational Science and \\ Engineering, EPFL - Ecole Polytechnique Fédérale de Lausanne, Station 8, CH-1015 Lausanne, Switzerland. \\ ${ }^{2}$ MOX - Modellistica e Calcolo Scientifico, Dipartimento di Matematica "F. Brioschi" - Politecnico di Milano, \\ P.za Leonardo da Vinci 32, I-20133 Milano, Italy. \\ ${ }^{3}$ SISSA Mathlab, International School for Advanced Studies, via Bonomea 265, I-34136 Trieste, Italy.
}

\section{SUMMARY}

The solution of inverse problems in cardiovascular mathematics is computationally expensive. In this paper we apply a domain parametrization technique to reduce both the geometrical and computational complexity of the forward problem, and replace the finite element solution of the incompressible NavierStokes equations by a computationally less expensive reduced basis approximation. This greatly reduces the cost of simulating the forward problem. We then consider the solution of inverse problems in both the deterministic sense, by solving a least-squares problem, and in the statistical sense, by using a Bayesian framework for quantifying uncertainty. Two inverse problems arising in haemodynamics modelling are considered: (i) a simplified fluid-structure interaction model problem in a portion of a stenosed artery for quantifying the risk of atherosclerosis by identifying the material parameters of the arterial wall based on pressure measurements; (ii) a simplified femoral bypass graft model for robust shape design under uncertain residual flow in the main arterial branch identified from pressure measurements. Copyright (c) 2013 John Wiley \& Sons, Ltd.

Received ..

KEY WORDS: Inverse problems; model reduction; shape optimization; fluid-structure interaction; reduced basis methods; haemodynamics; parametrized Navier-Stokes equations.

\section{INTRODUCTION}

Quite often computational models depend on uncertain elements, such as (i) material parameters and coefficients, boundary conditions, and (ii) geometrical (often patient-specific) configurations. Usually such factors cannot be completely identified to the point of absolute certainty; the former may be recovered from measurements, while the latter can be obtained as a result of a shape identification process. In general, inverse identification problems entail very large computational efforts, since they involve iterative optimization procedures that require several input/output evaluations. Incorporating geometrical configurations into the framework, e.g. when dealing with problems of fluid-structure interaction or optimal shape design, makes the inverse problem even less affordable.

Given a parametrized PDE model of our system, the forward problem consists in evaluating outputs of interest (depending on the state solution of the PDE) for specified parameter inputs.

†E-mail: grozza@sissa.it

*Correspondence to: Gianluigi Rozza, SISSA Mathlab, International School for Advanced Studies, via Bonomea 265,

I-34136 Trieste, Italy. 
On the other hand, whenever some parameters are uncertain, we aim at inferring their values (and/or distributions) from indirect observations (and/or measures) by solving an inverse problem: given an observed output, can we deduce the value of the parameters that resulted in this output? Such problems are often encountered in cardiovascular mathematics as problems of parameter identification $[7,70]$, variational data assimilation $[13,50,63,66]$, or shape optimization $[1,44,51,64]$. Computational inverse problems are characterized by two main difficulties:

1. The forward problem is typically a nonlinear PDE, possibly incorporating coupled multiphysics phenomena. State-of-the-art discretization methods and parallelized codes are therefore required to solve them up to a tolerable accuracy. This is exacerbated by the fact that solving the inverse problem requires multiple solutions of the forward problem. Hence, if the forward problem can be replaced with an inexpensive (but reliable) surrogate, solving the inverse problem is much more feasible.

2. Uncertainty in the model parameters can be large when the parameters describe geometric quantities such as shape. This is especially true in biomedical applications, where observed geometries are often patient-specific and only observable through medical imaging procedures, highly susceptible to measurement noise. In order to solve inverse problems in the presence of measurement errors it is typical to take advantage of some type of regularization (e.g. Tykhonov regularization) to recover existence and uniqueness of solutions.

Previous works on using parametric reduced order models to effectively solve computational inverse problems have exploited either the deterministic [72], the frequentist [26], or the Bayesian approach [20, 22]. We also mention strategies for dealing with nonstationary inverse problems based on the extended Kalman filtering approach [7, 40]. In general, largest computational gains are realized when the forward problem has to be solved for thousands of times on different parametric configurations. While an inverse problem (after regularization) can in principle be solved uniquely - for example with the classical maximum likelihood point estimator - providing single-valued parameter estimates, which minimize e.g. the defect between the observation and the forward model, is often deemed insufficient. In fact, the variability of the physiological conditions between patients even within the same class of healthy patients is large, and thus to obtain robust results that can be generalized to a large corpus of cases, one is faced with the inherent fact that some of the parameters of the forward problem must contain a significant amount of uncertainty.

In this work we propose a general framework for computationally solving inverse problems using reduced basis (RB) methods, and apply it to some inverse identification problems in haemodynamics, introducing a proof of concept for potential clinical applications. The first problem we consider involves the efficient forward simulation of steady incompressible flows in a simplified stenosed artery accounting for fluid-structure interaction mechanism, and the inverse identification of the material parameters of the arterial wall from the measurement of the pressure drop between inflow and outflow sections. The second problem involves the efficient forward simulation and robust shape optimization of a femoro-popliteal bypass anastomosis given uncertainty in the residual flow through the occluded femoral artery. In this latter case, the inverse identification problem is the one of determining the residual flow from the measurement of the pressure drop. We consider both the deterministic inverse problem (typically defined by solving for the maximum likelihood estimator) as well as a Bayesian approach. In order to set a framework for simultaneously reducing both geometrical and computational complexity, some simplifications have been considered in the case studies presented. In fact, a realistic model for blood circulation should take into account $(i)$ the flow unsteadiness, (ii) the arterial wall deformability, described by suitable structural models [25] and possibly (iii) complex rheological model to characterize the aggregate nature of the blood [57] especially in small vessels and capillaries. In view of studying optimization and inverse problems related with blood flows, which entail the repeated simulation of flow equations, we cannot afford at the moment the solution of PDE models involving such complex features - computational costs would still be too prohibitive. For these reasons, in the applications discussed throughout this paper, we model blood flows adopting steady incompressible Navier-Stokes equations, by showing the robustness, practicality and versatility of the proposed combined framework. 
The work is structured as follows: in Sec. 2 we introduce the general abstract model for the forward and inverse problems. In Sec. 3 we tackle the geometric variability of the domain using suitable parametric deformation maps, which in this work is done with free-form deformations. In Sec. 4 we briefly recall the reduced basis method for parametric model reduction, discussing its accuracy and reliability. In Sec. 5 we cover the first problem of inverse haemodynamics in a stenosed artery with fluid-structure interaction effects, whereas in Sec. 6 a robust shape design problem of femoro-popliteal bypass anastomoses is considered. We comment about the treatment of the errors arising from a reduced-order model in the Bayesian framework in Sec. 7. Further, we provide a possible roadmap for future research and generalization in this field in Sec. 8. The Appendix is focused on the application of RB methods to parametrized Navier-Stokes equations.

\section{ABSTRACT PARAMETRIZED FORMULATION}

In this section we introduce the abstract formulation of the forward problem representing our system of interest, some classes of inverse problems, and two possible frameworks for their solution.

\subsection{Abstract formulation of forward problems}

We assume that our system of interest is described by a parametrized PDE, where the parameter vector $\boldsymbol{\mu}=(\boldsymbol{\pi}, \boldsymbol{\omega}) \in \mathcal{D} \subset \mathbb{R}^{P}$ consists of two parts: an input or control parameter $\boldsymbol{\pi} \in \mathcal{D}_{\boldsymbol{\pi}} \subset \mathbb{R}^{P_{\boldsymbol{\pi}}}$ and an uncertainty parameter $\boldsymbol{\omega} \in \mathcal{D}_{\boldsymbol{\omega}} \subset \mathbb{R}^{P_{\boldsymbol{\omega}}}$, such that $\mathcal{D}=\mathcal{D}_{\boldsymbol{\pi}} \times \mathcal{D}_{\boldsymbol{\omega}}$ and $P=P_{\boldsymbol{\pi}}+P_{\boldsymbol{\omega}}$. In particular, we consider control parameters $\pi$ characterizing the geometric configuration $\Omega_{o}=$ $\Omega_{o}(\boldsymbol{\pi})$, and uncertainty parameters $\boldsymbol{\omega}$ related to physical properties, boundary conditions, or sources. We focus on the case of nonlinear stationary parametrized PDEs, whose abstract form is

$$
\mathcal{A}_{o}\left(U_{o}(\boldsymbol{\mu}), W\right)+\mathcal{C}_{o}\left(U_{o}(\boldsymbol{\mu}), U_{o}(\boldsymbol{\mu}), W\right)=\mathcal{F}_{o}(W) \quad \forall W \in X\left(\Omega_{o}(\boldsymbol{\pi})\right) ;
$$

$X\left(\Omega_{o}(\boldsymbol{\pi})\right)$ is the state space defined on $\Omega_{o}(\boldsymbol{\pi}), \mathcal{A}_{o}: X\left(\Omega_{o}(\boldsymbol{\pi})\right) \times X\left(\Omega_{o}(\boldsymbol{\pi})\right) \rightarrow \mathbb{R}$ is a continuous and uniformly inf-sup stable bilinear form, $\mathcal{C}_{o}: X\left(\Omega_{o}(\boldsymbol{\pi})\right) \times X\left(\Omega_{o}(\boldsymbol{\pi})\right) \times X\left(\Omega_{o}(\boldsymbol{\pi})\right) \rightarrow \mathbb{R}$ is a continuous trilinear form, while $\mathcal{F}_{o}: X\left(\Omega_{o}(\boldsymbol{\pi})\right) \rightarrow \mathbb{R}$ is a continuous linear form.

Given a parameter vector $\boldsymbol{\mu} \in \mathcal{D}$, the forward problem consists in computing the state solution $U_{o}(\boldsymbol{\mu})$ solving the state equation and in evaluating the corresponding output of interest

$$
s_{o}(\boldsymbol{\mu}):=\mathcal{S}_{o}\left(U_{o}(\boldsymbol{\mu})\right)=\mathcal{L}_{o}\left(U_{o}(\boldsymbol{\mu})\right)+\mathcal{Q}_{o}\left(U_{o}(\boldsymbol{\mu}), U_{o}(\boldsymbol{\mu})\right),
$$

which is usually given by a linear and/or a quadratic functional of the state solution $U_{o}(\boldsymbol{\mu})$; here $\mathcal{Q}_{o}: X\left(\Omega_{o}(\boldsymbol{\pi})\right) \times X\left(\Omega_{o}(\boldsymbol{\pi})\right) \rightarrow \mathbb{R}$ is a continuous positive semidefinite bilinear form, while $\mathcal{L}_{o}: X\left(\Omega_{o}(\boldsymbol{\pi})\right) \rightarrow \mathbb{R}$ is a continuous linear form. The solution of the forward problem (1)-(2) for many different values of the parameter vector $\boldsymbol{\mu}$ is thus equivalent to exploring the graph of the observation operator $\boldsymbol{\mu} \mapsto s_{o}(\boldsymbol{\mu})$.

If the forward problem consists in solving (1) in order to predict the outcome of an experiment (by computing the state variable $U_{o}(\boldsymbol{\mu})$ and evaluating the output of interest $s_{o}\left(U_{o}(\boldsymbol{\mu})\right)$ ), in the inverse problems observed data or measurements $s_{o}^{*}$ are used to estimate unknown parameters $\boldsymbol{\mu}$ of the physical system. In the following subsections, we discuss both a deterministic framework and a statistical framework for the solution of different types of inverse problems.

\subsection{A deterministic framework for inverse problems}

A deterministic approach for the solution of the inverse problems recasts them in a more general optimization framework, and computes the best-fit for the parameter value by minimizing the error (usually in the least squares sense) between the observation $s_{o}^{*}$ and the output prediction $s_{o}(\boldsymbol{\mu})$ given by the model. In our context based on parametrized PDEs, we define the abstract parametric inverselike problem as follows: given an observation operator $s_{o}: \mathcal{D} \rightarrow Y$, and a target observation $s_{o}^{*} \in Y$, find the parameter vector $\boldsymbol{\mu}^{*} \in \mathcal{D}$ that solves the optimization problem

$$
\boldsymbol{\mu}^{*}=\arg \min _{\boldsymbol{\mu} \in \mathcal{D}} J_{o}(\boldsymbol{\mu}):=\frac{1}{2}\left\|s_{o}^{*}-s_{o}(\boldsymbol{\mu})\right\|_{Y}^{2}+\frac{1}{2}\left\|\boldsymbol{\mu}-\boldsymbol{\mu}_{p}\right\|_{M}^{2} .
$$


Here $\boldsymbol{\mu}_{p} \in \mathcal{D}$ is a given, target parameter value, $M: \mathbb{R}^{P} \rightarrow \mathbb{R}^{P}$ is a positive definite matrix so that $\left\|\boldsymbol{\mu}-\boldsymbol{\mu}_{p}\right\|_{M}^{2}:=\left(M\left(\boldsymbol{\mu}-\boldsymbol{\mu}_{p}\right), \boldsymbol{\mu}-\boldsymbol{\mu}_{p}\right)_{\mathbb{R}^{P}}$ is a regularization term, $Y$ is the vector space of observables, $s_{o}(\boldsymbol{\mu})=\mathcal{S}_{o}\left(U_{o}(\boldsymbol{\mu})\right)$ is given by (2) and $U_{o}(\boldsymbol{\mu})$ solves the forward problem (1). The formulation (3) covers a large number of practical problems, of which we next give a few examples:

- Optimal design problem (without uncertainty)

$$
\min _{\boldsymbol{\pi} \in \mathcal{D}_{\boldsymbol{\pi}}} J_{o}(\boldsymbol{\pi}):=\frac{1}{2}\left\|\mathcal{S}_{o}\left(U_{o}(\boldsymbol{\pi})\right)\right\|_{Y}^{2},
$$

where the state equations (1) do not contain any uncertainty $\boldsymbol{\omega}$. We can also consider a tracking-type functional

$$
\min _{\boldsymbol{\pi} \in \mathcal{D}_{\boldsymbol{\pi}}} J_{o}(\boldsymbol{\pi}):=\frac{1}{2}\left\|s_{o}^{*}-\mathcal{S}_{o}\left(U_{o}(\boldsymbol{\pi})\right)\right\|_{Y}^{2},
$$

where $s_{o}^{*}=\mathcal{S}_{o}^{*}\left(U_{o}^{\text {ref }}\right)$ is the output related to a reference solution $U_{o}^{\text {ref }}$ with some desirable properties. For example, in a shape optimization problem related to minimizing the vortices generated behind a bluff body, $U_{o}^{\text {ref }}$ represent the Stokes solution (see e.g. [30, 34]).

- Robust optimal design problem (with uncertainty)

$$
\min _{\boldsymbol{\pi} \in \mathcal{D}_{\pi}} J_{o}(\boldsymbol{\pi}):=\max _{\boldsymbol{\omega} \in \mathcal{D}_{\boldsymbol{\omega}}} \frac{1}{2}\left\|\mathcal{S}_{o}\left(U_{o}(\boldsymbol{\mu})\right)\right\|_{Y}^{2},
$$

where we seek a single optimal shape represented through a parameter vector $\boldsymbol{\pi}^{*}$ to cover a range of possible realizations of the uncertainty $\boldsymbol{\omega}$ (thus considering a worst case scenario). This leads to a min-max optimization type problem:

$$
\min _{\boldsymbol{\pi} \in \mathcal{D}_{\boldsymbol{\pi}}} \max _{\boldsymbol{\omega} \in \mathcal{D}_{\boldsymbol{\omega}}} \frac{1}{2}\left\|\mathcal{S}_{o}\left(U_{o}(\boldsymbol{\mu})\right)\right\|_{Y}^{2} .
$$

- Parameter identification problem

$$
\min _{\boldsymbol{\omega} \in \mathcal{D}_{\boldsymbol{\omega}}} J_{o}(\boldsymbol{\mu}):=\frac{1}{2}\left\|s_{o}^{*}-\mathcal{S}_{o}\left(U_{o}\left(\boldsymbol{\pi}^{*}(\boldsymbol{\omega})\right)\right)\right\|_{Y}^{2}+\frac{1}{2}\left\|\boldsymbol{\omega}-\boldsymbol{\omega}_{p}\right\|_{M}^{2},
$$

where for any realization $\boldsymbol{\omega} \in \mathcal{D}_{\boldsymbol{\omega}}$ in addition to the forward problem

$$
\mathcal{A}_{o}\left(U_{o}\left(\boldsymbol{\pi}^{*}, \boldsymbol{\mu}\right), W\right)+\mathcal{C}_{o}\left(U_{o}\left(\boldsymbol{\pi}^{*}, \boldsymbol{\mu}\right), U_{o}\left(\boldsymbol{\pi}^{*}, \boldsymbol{\mu}\right), W\right)=\mathcal{F}_{o}(W) \quad \forall W \in X\left(\Omega_{o}\left(\boldsymbol{\pi}^{*}\right)\right),
$$

we need another equation, $\mathcal{G}_{o}\left(\boldsymbol{\pi}^{*}, \boldsymbol{\omega}\right)=0$, in order to close the problem and determine $\boldsymbol{\pi}^{*}=\boldsymbol{\pi}^{*}(\boldsymbol{\omega})$. For example, in an inverse fluid-structure interaction problem this yields to the solution for the structural displacement $\boldsymbol{\pi}^{*}$ given the material parameters $\boldsymbol{\omega}$ (see e.g. [50]).

Under the assumption that the control parameter vector $\boldsymbol{\mu} \in \mathcal{D} \subset \mathbb{R}^{P}$ is finite-dimensional, we have access to many tools from the theory of nonlinear programming [47]. Typically, we follow the direct method - solving directly the minimization problem by seeking a sequence $\left\{\boldsymbol{\mu}^{(k)}\right\}_{k=1,2, \ldots}$ values of $\boldsymbol{\mu}$, on which the value of the cost functional $J_{o}$ is reduced. Provided that the analytical expression of the first-order sensitivities $d J_{o} / d \mu_{j}$ are available, optimization can then be efficiently performed using affine-scaling interior-point Newton methods [9] for nonlinear programming with box constraints. The Hessian of the problem is replaced with quasi-Newton approximations, such as the BFGS method. For pure least-squares type functionals, there also exists the possibility of using the efficient Levenberg-Marquardt-Fletcher [18] method. The alternative would be indirect methods, i.e. solving the first order Karush-Kuhn-Tucker (KKT) necessary conditions (usually involving the solution of an adjoint problem). Due to the fact that the cost functional $J_{o}(\boldsymbol{\mu})$ depends on the solution of the state problem, it can be nonconvex and contain several local minima. Large-scale trust-region methods can help achieve global convergence of the optimization method [10]. Moreover, in the case of a min-max optimization problem of the type (7) we either need to solve an inner maximization problem inside each outer minimization problem, or consider a relaxation

$$
\min _{\boldsymbol{\pi} \in \mathcal{D}_{\boldsymbol{\pi}}} \max _{i=1, \ldots, n} \frac{1}{2}\left\|\mathcal{S}_{o}\left(U_{o}\left(\boldsymbol{\pi}, \boldsymbol{\omega}^{i}\right)\right)\right\|_{Y}^{2}
$$

for a discrete set of uncertainty values $\omega^{1}, \ldots, \boldsymbol{\omega}^{n} \in \mathcal{D}_{\boldsymbol{\omega}}$, after which the inner maximization reduces to a procedure of enumeration. 
No matter which numerical algorithm ends up being used, a deterministic setting for inverse problems - as well as optimal control or shape optimization problems - leads to the repeated evaluation of the state solution $U_{o}(\boldsymbol{\mu})$ and the cost functional $J_{o}(\boldsymbol{\mu})$, depending on the output of interest $\mathcal{S}_{o}\left(U_{o}(\boldsymbol{\mu})\right)$, which is a typical many-query problem. Hence, the efficient solution of (3) hinges on our ability to simulate effectively the forward problem (1): reduction techniques are thus mandatory in order to contain the computational costs, since optimization procedures may require up to $\mathcal{O}\left(10^{2}\right)-\mathcal{O}\left(10^{3}\right)$ input/output evaluations.

Further difficulties are exhibited when the parameters $\pi$ control explicitly or implicitly the domain of the forward problem $\Omega_{o}(\boldsymbol{\pi})$, as in our case. When the forward problem is approximated using e.g. the finite element or finite volume method, changing the underlying geometry requires an expensive and involved mesh deformation or remeshing processes, followed by the re-assembling of the entire linear system. Since in large-scale inverse-like problems this is too costly, we consider only fixed domain approaches, where the family of admissible domains $\Omega_{o}(\boldsymbol{\pi})$ is given as the image of a smooth parametric map

$$
T(\cdot ; \boldsymbol{\pi}): \Omega \rightarrow \Omega_{o}(\boldsymbol{\pi}) .
$$

In the next section we describe a specific technique for obtaining parametric geometric maps $T$ which have good properties in combination with model reduction strategies, namely the free-form deformation technique [36, 38, 44, 60]. Hence, both the solution of the forward problem and the observations can be transformed by a change of variables to the fixed reference domain $\Omega$, resulting in the inverse-like problem

$$
\min _{\boldsymbol{\mu} \in \mathcal{D}_{\boldsymbol{\mu}}} J(\boldsymbol{\mu}):=\frac{1}{2}\left\|s^{*}-s(\boldsymbol{\mu})\right\|_{Y}^{2}+\frac{1}{2}\left\|\boldsymbol{\mu}-\boldsymbol{\mu}_{p}\right\|_{M}^{2},
$$

where $s(\boldsymbol{\mu})=\mathcal{S}(U(\boldsymbol{\mu}) ; \boldsymbol{\mu})$ and $U(\boldsymbol{\mu})$ is the solution of the forward problem on the fixed domain:

$$
\mathcal{A}(U(\boldsymbol{\mu}), W ; \boldsymbol{\mu})+\mathcal{C}(U(\boldsymbol{\mu}), U(\boldsymbol{\mu}), W ; \boldsymbol{\mu})=\mathcal{F}(W ; \boldsymbol{\mu}) \quad \forall W \in X(\Omega) .
$$

Here $X(\Omega)$ is a suitable functional space defined on the reference domain $\Omega, \mathcal{A}: X(\Omega) \times X(\Omega) \rightarrow$ $\mathbb{R}$ is a parametrized, continuous and inf-sup stable bilinear form, $\mathcal{C}: X(\Omega) \times X(\Omega) \times X(\Omega) \rightarrow \mathbb{R}$ is a parametrized, continuous trilinear form, while $\mathcal{F}: X(\Omega) \rightarrow \mathbb{R}$ is a parametrized, continuous linear form. This formulation can be obtained from (1)-(2) by means of a change of variables; the case of Navier-Stokes equations, employed in the applications, will be detailed in the Appendix A.

Once the problem has been rewritten in the proposed parametrized form (11)-(12), thanks to the assumption of affine parametric dependence (see Sec. 4) we can exploit the RB method to speed up the solution of the state problem and related output evaluations during an optimization procedure.

\subsection{A statistical framework for inverse problems}

As pointed out in the previous subsection, a deterministic framework for inverse problems provides, through the solution of a suitable optimization problem, the best estimator of the parameter vector $\boldsymbol{\mu}^{*}$ given some data or measurements $s^{*}$; often suitable regularization is needed [29] in order to remove the ill-posedness of the problem and to evaluate the inverse map $s^{*} \mapsto \boldsymbol{\mu}^{*}\left(s^{*}\right)$. In the case that $s^{*}$ is an experimental measure, possibly polluted by measurement error, a deterministic approach is no longer sufficient to describe fully the propagation of errors through the numerical model. Moreover, we need a method for quantifying the uncertainty contained in the best estimator. For these reasons we turn to a statistical framework, which is able to provide an uncertainty quantification on the estimated parameter values that are described by a suitable probability distribution [45]. We remark that the statistical approach to uncertainty in inverse problems, either from the frequentist [56] or the Bayesian viewpoint [29, 67], requires in any case the repeated evaluation of outputs over large sample sets (e.g. in order to compute sample statistics such as expectations, variances, and higher moments). Here we do not treat in detail statistical inverse problems and the various approaches that have been proposed in what is a vast field of applied statistics, but limit ourselves to mention that $(i)$ reduction techniques prove to be mandatory also within a statistical approach (as detailed in [20, 22, 39]) and that (ii) the reduced basis framework is suitable also for uncertainty quantification [26] and more general probabilistic problems [8,46]. 
In this paper we identify two approaches to deal with uncertainty. In the first approach, we aim at quantifying how noise sources affect the predicted quantities of the simulations, so that some application-specific conclusions can be drawn. In order to facilitate such an approach, we assume that the uncertainty can be embedded in the parameters of the forward problem - we do not consider nonparametric approaches here $\mathrm{b}^{\mathrm{b}}$ - and that the parametric dimension is relatively small to avoid the curse of dimensionality. Exploring the parametric uncertainty space of possible outcomes leads naturally to a "many-query" context - the forward problem will need to be solved for multiple different values of the uncertainty parameters to either arrive at a full exploration of possible outcomes or at least the most likely ones up to some confidence interval.

In the second approach a Bayesian viewpoint (see e.g. [22, 39]) is adopted, providing a probability distribution function for the parameter $\boldsymbol{\mu}$ that carries on the uncertainties related to measurements. Within this framework, we model observations $s^{*}$ and parameters $\boldsymbol{\mu}$ as random variables with an associated probability density function. The solution of the inverse problem is given within this framework by the posterior probability density $p\left(\boldsymbol{\mu} \mid s^{*}\right): \mathcal{D}_{\boldsymbol{\mu}} \times Y \rightarrow \mathbb{R}_{0}^{+}$, i.e. the probability density of the parameter $\boldsymbol{\mu}$ given the measured value of $s^{*}$, which can be obtained by the Bayes' theorem

$$
p_{\boldsymbol{\mu} \mid s^{*}}\left(\boldsymbol{\mu} \mid s^{*}\right)=\frac{p_{s^{*} \mid \boldsymbol{\mu}}\left(s^{*} \mid \boldsymbol{\mu}\right) p_{\boldsymbol{\mu}}(\boldsymbol{\mu})}{p_{s^{*}}\left(s^{*}\right)},
$$

where, in (13), $p_{\boldsymbol{\mu}}: \mathcal{D}_{\boldsymbol{\mu}} \rightarrow \mathbb{R}_{0}^{+}$is the prior probability density, expressing all available information on $\boldsymbol{\mu}$ independently of the particular measurements on $s^{*}$ that will be considered as data; $p_{s^{*} \mid \mu}$ : $Y \times \mathcal{D}_{\mu} \rightarrow \mathbb{R}_{0}^{+}$is the likelihood function, i.e. the conditional probability density of the observation $s^{*}$ if $\boldsymbol{\mu}$ were known; $p_{s^{*}}(\cdot)$ is a norming constant, determined by the normalization of the posterior density, and has often little importance. In particular, we consider an additive noise model, so that

$$
s^{*}=s(\boldsymbol{\mu})+\varepsilon,
$$

and our data are $n$ noised measures modelled as in (14). This procedure is quite common in the analysis of statistical inverse problems (see e.g. [3, 29]). The simplest probabilistic model that can be used to describe experimental uncertainties is the Gaussian model: under this assumption ${ }^{\mathrm{c}}$ the likelihood function is given instead by

$$
p_{s^{*} \mid \boldsymbol{\mu}}\left(\mathbf{s}^{*} \mid \boldsymbol{\mu}\right) \propto \exp \left(-\frac{1}{2}\left\|\mathbf{s}^{*}-\mathbf{s}(\boldsymbol{\mu})\right\|_{\Sigma_{s}^{-1}}^{2}\right)=\exp \left(-\frac{1}{2 \sigma^{2}}\left\|\mathbf{s}^{*}-\mathbf{s}(\boldsymbol{\mu})\right\|^{2}\right) .
$$

If we can assume a Gaussian model also on the prior knowledge of the parameters distributions, i.e. $p_{\boldsymbol{\mu}}(\boldsymbol{\mu}) \sim \mathcal{N}\left(\boldsymbol{\mu}_{p}, \Sigma_{p}\right)$, then the posterior probability density will be normally distributed, i.e.

$$
p_{\boldsymbol{\mu} \mid s^{*}}\left(\boldsymbol{\mu} \mid \mathbf{s}^{*}\right) \propto \exp \left(-\frac{1}{2}\left\|\mathbf{s}^{*}-\mathbf{s}(\boldsymbol{\mu})\right\|_{\Sigma_{s}^{-1}}^{2}-\frac{1}{2}\left\|\boldsymbol{\mu}-\boldsymbol{\mu}_{p}\right\|_{\Sigma_{p}^{-1}}^{2}\right) .
$$

In this case, the maximum a posteriori estimator $\boldsymbol{\mu}^{*}$, obtained by maximizing the posterior distribution (15), is

$$
\boldsymbol{\mu}^{*}=\arg \min _{\boldsymbol{\mu} \in \mathbb{R}^{p}}\left(\frac{1}{2}\left\|\mathbf{s}^{*}-\mathbf{s}(\boldsymbol{\mu})\right\|_{\Sigma_{s}^{-1}}^{2}+\frac{1}{2}\left\|\boldsymbol{\mu}-\boldsymbol{\mu}_{p}\right\|_{\Sigma_{p}^{-1}}^{2}\right),
$$

i.e. by solving a specific instance of the regularized minimization problem (3). We can also remark that in the Bayesian framework the norms $\|\cdot\|_{Y},\|\cdot\|_{M}$ and the value $\boldsymbol{\mu}_{p}$ have a clear interpretation in terms of the statistics of the measurement noise and the prior probability distribution [67].

\footnotetext{
${ }^{b}$ We call the subset of parameters that describe the uncertainty as uncertainty parameters, as opposed to the other types of parameters in our inverse problem called control parameters that can be either directly or indirectly influenced.

${ }^{c}$ In other words, we have normally distributed errors $\varepsilon \sim \mathcal{N}\left(\mathbf{0}, \Sigma_{s}\right)$, uncorrelated and equally distributed, i.e. $\Sigma_{s}=\sigma^{2} \mathbb{I}_{n}$, where the variance $\sigma^{2}$ is supposed to be known and independent of $\boldsymbol{\mu}$.
} 
On the other hand, if we assume that no information is available about the parameter distribution except that it resides in the parameter space $\mathcal{D}$, i.e. $p_{\boldsymbol{\mu}}(\boldsymbol{\mu}) \sim \mathcal{U}(\mathcal{D})$ is a uniform distribution over $\mathcal{D}$, the posterior probability density is given by

$$
p_{\boldsymbol{\mu} \mid s^{*}}\left(\boldsymbol{\mu} \mid \mathbf{s}^{*}\right) \propto \begin{cases}\exp \left(-\frac{1}{2 \sigma^{2}}\left\|\mathbf{s}^{*}-\mathbf{s}(\boldsymbol{\mu})\right\|^{2}\right), & \boldsymbol{\mu} \in \mathcal{D}, \\ 0, & \boldsymbol{\mu} \notin \mathcal{D} .\end{cases}
$$

If the parameter space $\mathcal{D}$ has a large dimension (e.g. $P>4$ ), an exhaustive exploration of this space is not possible, and we have to rely on Monte Carlo sampling techniques to extract information from the posterior probability density; a well-known technique for sampling probability distributions is the Metropolis-Hastings algorithm, which proceeds as follows:

1. Pick an initial $\boldsymbol{\mu}^{1}$.

2. For $k=1, \ldots, K$ :

(a) Compute $p_{\boldsymbol{\mu} \mid s^{*}}\left(\boldsymbol{\mu}^{k} \mid s^{*}\right)$.

(b) Take a random step to find the next candidate $\widehat{\boldsymbol{\mu}}:=\boldsymbol{\mu}^{k}+\mathcal{N}(0, \Delta I)$.

(c) Compute $p_{\boldsymbol{\mu} \mid s^{*}}\left(\widehat{\boldsymbol{\mu}} \mid s^{*}\right)$.

(d) Define the acceptance ratio $\alpha_{k}:=\min \left\{1, \frac{p_{\boldsymbol{\mu} \mid s^{*}}\left(\widehat{\boldsymbol{\mu}} \mid s^{*}\right)}{p_{\boldsymbol{\mu} \mid s^{*}}\left(\boldsymbol{\mu}^{k} \mid s^{*}\right)}\right\}$.

(e) Let $u \sim \mathcal{U}([0,1])$; if $u \leq \alpha_{k}$, accept, set $\boldsymbol{\mu}^{k+1}=\widehat{\boldsymbol{\mu}}$, and add to the set of samples $\Xi$. Otherwise reject, and keep $\boldsymbol{\mu}^{k+1}=\boldsymbol{\mu}^{k}$.

After the algorithm has taken $K$ steps, the sample set $\Xi$ contains realizations of the probability distribution $p_{\boldsymbol{\mu} \mid s^{*}}\left(\boldsymbol{\mu} \mid s^{*}\right)$. The diagonal values of the step size matrix $\Delta$ should be chosen as large as possible while still maintaining a reasonable acceptance rate [29].

We point out that the continuous model giving the output $s(\boldsymbol{\mu})$ is never computable in practice, and will always be replaced by a quantity which can be evaluated. For instance, we could discretize the forward model (12) by means of a high-fidelity finite element approximation, and denote as $s_{h}(\boldsymbol{\mu})$ the corresponding output. Indeed, we consider a further step, since we rely on a reduced model built over a finite element approximation. Its construction is detailed in Sect. 4, while its impact on the solution of an inverse problem is largely discussed in Sect. 7.

\section{GEOMETRICAL REDUCTION TECHNIQUES}

Both fluid-structure interaction (FSI) and shape optimization problems deal traditionally with (i) discretization techniques over domains of variable shape; (ii) mesh motion and possibly remeshing during iterative procedures for coupling or optimization; and (iii) the need of exchanging information over the free boundary (either by transferring loads and displacements between fluid and structure, or moving the fluid boundary according to the minimization of a cost functional):

- In FSI problems, dealt with by splitting iterative procedures, stresses or pressure loads are transmitted from the fluid domain to the structural finite element nodes sitting on the fluidstructure interface. Once the structure motion is determined, the displacement of the fluid mesh points on the interface has to be imposed in order to take into account the structural deformation rate and the new computational domain of the fluid;

- In shape optimization problems, the nodes lying on the boundary to be optimized are usually displaced according to the shape gradient of the chosen cost functional to be minimized.

In order to apply the reduced basis methods for an efficient solution of the fluid equations, we need to rely on a fixed domain approach and a suitable shape parametrization technique for describing a family of admissible shape configurations by mapping a reference ( $\pi$-independent) domain $\Omega$. 
In this way, basis solutions corresponding to different shape configurations $\Omega_{o}(\boldsymbol{\pi})$ can be compared and combined. Not only, if the parametric map $T(\cdot ; \boldsymbol{\pi}): \Omega \rightarrow \Omega_{o}(\boldsymbol{\pi})$ is sufficiently flexible and low-dimensional, the introduction of a parametrized map can be seen as a first reduction step, since computations can be performed on the reference configuration and shape deformation can be easily handled by acting on a small number of geometrical parameters.

Different options for the construction of the parametric map $T(\cdot ; \boldsymbol{\pi})$ have been considered in the reduced basis framework. For example, affine or nonaffine maps based on a domain decomposition approach have been introduced in order to deal with simple cartesian geometries; several extensions to geometries with curved boundaries have been also implemented within the rbMIT library exploited for RB computations [27, 59], but leading sometimes to large parametric complexities (in terms of piecewise local affine expansions of the operators) when dealing with more involved geometries. An alternative way for constructing flexible but low-dimensional maps is based on the so-called free shape representations, which are obtained by introducing a small set of control points (and possibly suitable interpolation strategies) whose possible displacements induce the shape deformation and represent the geometrical parameters, rather than geometrical properties themselves. The most popular technique within this group is the free-form deformation (FFD) technique, which is based on tensor products of splines and a global nonaffine transformation map.

The free-form deformation technique was introduced in [65]. Here we describe its simpler (and more classical) version ${ }^{\mathrm{d}}$, which will be subsequently exploited in the example discussed in Sec. 6 . Let $\Omega \subset D$ be embedded inside a fixed "holdall" parallelogram $D$, which can be mapped affinely onto the unit square, i.e. $\Psi(D)=(0,1) \times(0,1)$ with coordinates $\hat{\boldsymbol{x}}=\left(\hat{x}_{1}, \hat{x}_{2}\right)$. We introduce on the unit square a regular grid of $(L+1) \times(M+1)$ control points

$$
\mathbf{P}_{l, m}=[l / L, m / M]^{T}, \quad l=0, \ldots, L, \quad m=0, \ldots, M
$$

whose possible deformations are specified by a set of $(L+1)(M+1)$ parameter vectors $\pi_{l, m} \in \mathbb{R}^{2}$, such that the corresponding perturbed control points are given by

$$
\mathbf{P}_{l, m}^{o}\left(\boldsymbol{\pi}_{l, m}\right)=\mathbf{P}_{l, m}+\boldsymbol{\pi}_{l, m} .
$$

Then we can define the parametric map $\hat{T}(\cdot ; \boldsymbol{\pi}): \hat{D} \rightarrow \hat{D}_{o}(\boldsymbol{\pi})$ as

$$
\hat{T}(\hat{\boldsymbol{x}} ; \boldsymbol{\pi})=\left(\sum_{l=0}^{L} \sum_{m=0}^{M} b_{l, m}^{L, M}(\hat{\boldsymbol{x}}) \mathbf{P}_{l, m}^{o}\left(\boldsymbol{\pi}_{l, m}\right)\right),
$$

where

$$
b_{l, m}^{L, M}(\hat{\boldsymbol{x}})=b_{l}^{L}\left(\hat{x}_{1}\right) b_{m}^{M}\left(\hat{x}_{2}\right)=\left(\begin{array}{c}
L \\
l
\end{array}\right)\left(\begin{array}{c}
M \\
m
\end{array}\right)\left(1-\hat{x}_{1}\right)^{L-l} \hat{x}_{1}^{l}\left(1-\hat{x}_{2}\right)^{M-m} \hat{x}_{2}^{m}
$$

are tensor products of the unidimensional Bernstein basis polynomials defined on $\hat{D}$ by

$$
b_{l}^{L}\left(\hat{x}_{1}\right)=\left(\begin{array}{c}
L \\
l
\end{array}\right) \hat{x}_{1}^{l}\left(1-\hat{x}_{1}\right)^{L-l}, \quad b_{m}^{M}\left(\hat{x}_{2}\right)=\left(\begin{array}{c}
M \\
m
\end{array}\right) \hat{x}_{2}^{m}\left(1-\hat{x}_{2}\right)^{M-m}
$$

and forming a total of $(L+1)(M+1)$ basis polynomials. Finally, the FFD mapping $T(\cdot, \boldsymbol{\pi})$ is obtained as the composition

$$
T(\cdot ; \boldsymbol{\pi}): D \rightarrow D_{\circ}(\boldsymbol{\pi}), \quad T(\boldsymbol{x} ; \boldsymbol{\pi})=\Psi^{-1} \circ \hat{T} \circ \Psi(\boldsymbol{x} ; \boldsymbol{\pi}) ;
$$

in particular, the parametrized domain $\Omega_{o}(\boldsymbol{\pi})$ is obtained as $\Omega_{o}(\boldsymbol{\pi})=\Psi^{-1} \circ \hat{T} \circ \Psi(\Omega ; \boldsymbol{\pi})$. In Fig. 1 we display a schematic diagram of the FFD mapping construction.

${ }^{d}$ FFD parametrizations can be built by considering more complex basis functions instead of Bernstein polynomials (also denoted as Bézier parametrization case): extensions of FFD to Non-Uniform and Rational B-Splines (NURBS) basis functions are also available (see e.g. [2, 21]). 


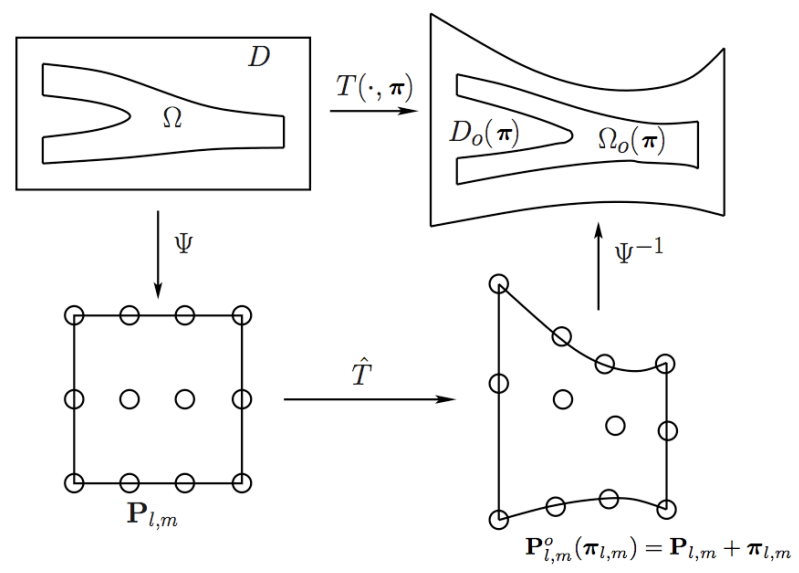

Figure 1. Schematic diagram of the FFD technique: unperturbed control points $\mathbf{P}_{l, m}$, perturbed control points $\mathbf{P}_{l, m}^{o}\left(\boldsymbol{\pi}_{l, m}\right)$, map $\hat{\boldsymbol{x}}=\Psi(\boldsymbol{x}), \hat{T}(\hat{\boldsymbol{x}} ; \boldsymbol{\pi})$ and resulting FFD map $T(\boldsymbol{x} ; \boldsymbol{\pi})=\left(\Psi^{-1} \circ \hat{T} \circ \Psi\right)(\boldsymbol{x} ; \boldsymbol{\pi})$.

Using the definition (17) and the fact that the Bernstein polynomials form a partition of unity, it can be simply shown that free-form deformations are a particular perturbation of the identity, i.e. $T(\boldsymbol{x} ; \mathbf{0})=\boldsymbol{x}$. In case there is a need to reduce the number of geometrical parameters, we can keep fixed a subset of control points or only allow them to move in one direction; this allows the user to keep the number of FFD parameters to a desired low level (in our case $P<10$ is typical). Adaptive procedures for the selection of the control points based on sensitivity analysis and correlations are also available. In the next section we introduce the basic ingredients for computational reduction by means of the reduced basis methods in order to approximate in a rapid and reliable way solutions to (12) and then evaluate the parametric output (11).

\section{COMPUTATIONAL REDUCTION TECHNIQUES}

Computing an approximate solution of (possibly nonlinear) PDE systems may be very expensive with respect to both CPU time and storage whenever standard discretization techniques are used. In particular, it is very difficult to deal with many-query and real-time situations, such as parametric analyses, optimal control problems, or rapid input-output evaluations. For this reason, reducing the costs of state solutions and output evaluations plays a fundamental role in many applications and has spurred the development of several reduced-order strategies for nonlinear problems.

In the reduced basis method $[49,59]$ the solution of a given problem is expressed as a linear combination of global basis solutions; thus, it is made up by (i) the construction of a reduced basis (of solutions of the high-dimensional discrete PDE system, also called snapshots), (ii) the approximation of the solution as a linear combination of the reduced basis functions and (iii) the determination of the coefficients in the linear combination through the solution (by Galerkin projection) of the PDE problem in the reduced basis space. The first part requires several solutions of the full, high-dimensional problem, which are performed only once, during the so-called Offline stage; each Online evaluation of the reduced solution (and related output) thus requires the solution of a very reduced problem, whose cost is very small whence the dimension of the reduced basis is small (compared to the dimension of the full discretized problem).

In this section we introduce the main ingredients for the reduced basis approximation of the abstract inverse problem (11)-(12), rewritten here by dropping the nonlinear terms for the sake of simplicity:

$$
\begin{gathered}
\text { find } \hat{\boldsymbol{\mu}}=\arg \min _{\boldsymbol{\mu} \in \mathcal{D}} J(\boldsymbol{\mu}):=\left\|s^{*}-s(\boldsymbol{\mu})\right\|_{Y}^{2}+\frac{1}{2}\left\|\boldsymbol{\mu}-\boldsymbol{\mu}_{p}\right\|_{M}^{2} \text { s.t. } \\
s(\boldsymbol{\mu})=\mathcal{S}(U(\boldsymbol{\mu}) ; \boldsymbol{\mu}) \text {, with } U(\boldsymbol{\mu}) \in X(\Omega): \mathcal{A}(U(\boldsymbol{\mu}), W ; \boldsymbol{\mu})=\mathcal{F}(W ; \boldsymbol{\mu}), \forall W \in X(\Omega) .
\end{gathered}
$$

In particular, let us make the following assumptions: 
1. $\mathcal{A}(\cdot, \cdot ; \boldsymbol{\mu})$ is a continuous bilinear form such that the following uniform inf-sup stability condition is satisfied:

$$
\exists \beta_{0}>0: \beta_{0}<\beta(\boldsymbol{\mu}):=\inf _{V \in X} \sup _{W \in X} \frac{\mathcal{A}(V, W ; \boldsymbol{\mu})}{\|V\|_{X}\|W\|_{X}}, \forall \boldsymbol{\mu} \in \mathcal{D} ;
$$

$\mathcal{F}(\cdot ; \boldsymbol{\mu})$ is a continuous linear form for all $\boldsymbol{\mu} \in \mathcal{D}$.

2. $\mathcal{A}(\cdot, \cdot ; \boldsymbol{\mu})$ and $\mathcal{F}(\cdot ; \boldsymbol{\mu})$ satisfy the affine parametric dependence property, i.e. they can be written as a linear combination of parameter independent bilinear/linear forms as follows:

$$
\mathcal{A}(V, W ; \boldsymbol{\mu})=\sum_{q=1}^{Q_{\mathcal{A}}} \Theta_{q}^{\mathcal{A}}(\boldsymbol{\mu}) \mathcal{A}_{q}(V, W), \quad \mathcal{F}(W ; \boldsymbol{\mu})=\sum_{q=1}^{Q_{\mathcal{F}}} \Theta_{q}^{f}(\boldsymbol{\mu}) \mathcal{F}_{q}(W),
$$

for some integers $Q_{\mathcal{A}}, Q_{\mathcal{F}}$, which are an indication of the parametric complexity of the problem. If this assumption is not automatically fulfilled (for example, this is the case where the geometric mapping $T(\cdot ; \boldsymbol{\pi})$ is nonaffine), an approximate affine expansion like in (19) can be obtained through the so-called Empirical Interpolation Method (EIM) [6], as shown in $[38,36,44]$.

3. the output of interest $s(\boldsymbol{\mu})=\mathcal{S}(U(\boldsymbol{\mu}) ; \boldsymbol{\mu})$ is given by a quadratic functional of the state variable, i.e. $s(\boldsymbol{\mu})=\mathcal{L}(U(\boldsymbol{\mu}) ; \boldsymbol{\mu})+\mathcal{Q}(U(\boldsymbol{\mu}), U(\boldsymbol{\mu}) ; \boldsymbol{\mu})$, where $\mathcal{L}(\cdot ; \boldsymbol{\mu})$ and $\mathcal{Q}(\cdot, \cdot ; \boldsymbol{\mu})$ satisfy the same affine parametric dependence property:

$$
\mathcal{L}(W ; \boldsymbol{\mu})=\sum_{q=1}^{Q_{\mathcal{L}}} \Theta_{q}^{\mathcal{L}}(\boldsymbol{\mu}) \mathcal{L}_{q}(W), \quad \mathcal{Q}(V, W ; \boldsymbol{\mu})=\sum_{q=1}^{Q_{\mathcal{Q}}} \Theta_{q}^{\mathcal{Q}}(\boldsymbol{\mu}) \mathcal{Q}_{q}(V, W) .
$$

For the sake of simplicity, we shall assume that $s(\boldsymbol{\mu})=\mathcal{Q}(U(\boldsymbol{\mu}), U(\boldsymbol{\mu}) ; \boldsymbol{\mu})$, i.e. we will deal with a pure quadratic output in the rest of the section.

We start from the full, high-dimensional (or truth) approximation of the problem (11)-(12), which can be obtained by the standard Galerkin-Finite Element (FE) method:

$$
\begin{gathered}
\text { find } \hat{\boldsymbol{\mu}}=\arg \min _{\boldsymbol{\mu} \in \mathcal{D}_{a d}} J_{h}(\boldsymbol{\mu})=\left\|s^{*}-s_{h}(\boldsymbol{\mu})\right\|_{Y}^{2}+\frac{1}{2}\left\|\boldsymbol{\mu}-\boldsymbol{\mu}_{p}\right\|_{M}^{2} \text { s.t. } \\
s_{h}(\boldsymbol{\mu})=\mathcal{S}\left(U_{h}(\boldsymbol{\mu}) ; \boldsymbol{\mu}\right) \text {, with } U_{h}(\boldsymbol{\mu}) \in X_{h}: \mathcal{A}\left(U_{h}(\boldsymbol{\mu}), W ; \boldsymbol{\mu}\right)=\mathcal{F}(W ; \boldsymbol{\mu}), \forall W \in X_{h},
\end{gathered}
$$

where $X_{h}$ is a proper finite element space of dimension $\mathcal{N}_{h}=\operatorname{dim}\left(X_{h}\right)$. We assume that the FE space is chosen such that the state problem remains inf-sup stable, i.e.

$$
\exists \beta_{h, 0}>0: \beta_{0}<\beta_{h, 0}<\beta_{h}(\boldsymbol{\mu}):=\inf _{V \in X_{h}} \sup _{W \in X_{h}} \frac{\mathcal{A}(V, W ; \boldsymbol{\mu})}{\|V\|_{X}\|W\|_{X}}, \forall \boldsymbol{\mu} \in \mathcal{D} ;
$$

typical choices for the approximation of Stokes or Navier-Stokes problems are the Taylor-Hood $\mathbb{P}_{n} / \mathbb{P}_{n-1}$ couples of FE spaces for velocity and pressure, respectively (for $n \geq 2$ ).

The reduced basis method $[59,49,53]$ provides an efficient way to compute an approximation $U_{N}(\boldsymbol{\mu})$ of $U_{h}(\boldsymbol{\mu})$ (and related output) in the following way:

1. Construction of the reduced subspace. The reduced subspace $X_{N}$ is made up of well-chosen FE solutions, corresponding to a specific choice $S_{N}=\left\{\boldsymbol{\mu}^{1}, \ldots, \boldsymbol{\mu}^{N}\right\}$ of parameter values. This space is built by means of an adaptive, iterative greedy procedure [59], based on the a posteriori error bound introduced in the following. Let us denote $\Xi_{\text {train }} \subset \mathcal{D}$ a (sufficiently rich) finite training sample of parameter points chosen using a uniform distribution on $\mathcal{D}$. Hence, given the first parameter value $\mu^{1}$ and a sharp, rigorous and inexpensive error bound $\Delta_{N}(\boldsymbol{\mu})[49,59]$ such that, for the generic approximation $U_{N}(\boldsymbol{\mu}) \in X_{N}, N=1, \ldots, N_{\max }$,

$$
\left\|U_{h}(\boldsymbol{\mu})-U_{N}(\boldsymbol{\mu})\right\|_{\mathcal{Y}} \leq \Delta_{N}(\boldsymbol{\mu}) \text { for all } \boldsymbol{\mu} \in \Xi_{\text {train }},
$$


we choose the remaining parameter values (and corresponding snapshot solutions) as

$$
\boldsymbol{\mu}^{N}:=\arg \max _{\boldsymbol{\mu} \in \Xi_{\text {train }}} \Delta_{N-1}(\boldsymbol{\mu}), \quad \text { for } N=2, \ldots, N_{\max }
$$

until an error tolerance $\varepsilon_{t o l}^{R B}$ a priori fixed is achieved: $\Delta_{N}(\boldsymbol{\mu}) \leq \varepsilon_{t o l}^{R B}$ for all $\boldsymbol{\mu} \in \Xi_{\text {train }}$.

2. Reduced basis approximation. Denoting $X_{N}=\operatorname{span}\left\{U_{h}\left(\boldsymbol{\mu}^{N}\right), N=1, \ldots, N_{\max }\right\}$ the RB space, the RB approximation of (11)-(12) is obtained by means of a Galerkin projection onto the reduced space $X_{N}$ as follows:

$$
\begin{gathered}
\text { find } \hat{\boldsymbol{\mu}}=\arg \min _{\boldsymbol{\mu} \in \mathcal{D}_{a d}} J_{N}(\boldsymbol{\mu})=\left\|s^{*}-s_{N}(\boldsymbol{\mu})\right\|_{Y}^{2}+\frac{1}{2}\left\|\boldsymbol{\mu}-\boldsymbol{\mu}_{p}\right\|_{M}^{2} \text { s.t. } \\
s_{N}(\boldsymbol{\mu})=\mathcal{S}\left(U_{N}(\boldsymbol{\mu}) ; \boldsymbol{\mu}\right), \text { with } U_{N}(\boldsymbol{\mu}) \in X_{N}: \mathcal{A}\left(U_{N}(\boldsymbol{\mu}), W ; \boldsymbol{\mu}\right)=\mathcal{F}(W ; \boldsymbol{\mu}), \forall W \in X_{N} .
\end{gathered}
$$

By expressing the RB solution as $U_{N}(\boldsymbol{\mu})=\sum_{i=1}^{N} u_{i}(\boldsymbol{\mu}) \boldsymbol{\phi}_{i}$, being $\left\{\boldsymbol{\phi}_{i}\right\}_{i=1}^{N}$ an orthonormalized (by Gram-Schmidt) basis of the space $X_{N}$, the equivalent matricial formulation of problem (23) becomes:

$$
\text { find } \begin{aligned}
\hat{\boldsymbol{\mu}}=\arg \min _{\boldsymbol{\mu} \in \mathcal{D}_{a d}} J_{N}(\boldsymbol{\mu})= & \left\|s^{*}-\mathbf{U}_{N}(\boldsymbol{\mu})^{T} \mathbb{Q}_{N}(\boldsymbol{\mu}) \mathbf{U}_{N}(\boldsymbol{\mu})\right\|_{Y}^{2}+\frac{1}{2}\left\|\boldsymbol{\mu}-\boldsymbol{\mu}_{p}\right\|_{M}^{2} \text { s.t. } \\
& \mathbb{A}_{N}(\boldsymbol{\mu}) \mathbf{U}_{N}(\boldsymbol{\mu})=\mathbb{F}_{N}(\boldsymbol{\mu})
\end{aligned}
$$

where $\mathbf{U}(\boldsymbol{\mu})=\left(u_{1}(\boldsymbol{\mu}), \ldots, u_{N}(\boldsymbol{\mu})\right)^{T}$ and, for $1 \leq l, m \leq N$,

$$
\left(\mathbb{A}_{N}\right)_{l m}(\boldsymbol{\mu})=\mathcal{A}\left(\phi_{m}, \phi_{l} ; \boldsymbol{\mu}\right), \quad\left(\mathbb{F}_{N}\right)_{l}(\boldsymbol{\mu})=\mathcal{F}\left(\phi_{l} ; \boldsymbol{\mu}\right), \quad\left(\mathbb{Q}_{N}\right)_{l m}(\boldsymbol{\mu})=\mathcal{Q}\left(\phi_{m}, \boldsymbol{\phi}_{l} ; \boldsymbol{\mu}\right) .
$$

Thanks to the (considerably) reduced dimension $O(N) \ll O\left(\mathcal{N}_{h}\right)$ of the linear systems obtained from RB approximation, the cost of the solution of the RB state problem (and related iterative optimization procedure) is very small compared to that of the truth FE approximation.

3. Offline-Online decomposition. Under the affinity assumption (19)-(20), RB operators can be written isolating the parametric contribution as follows:

$$
\mathbb{A}_{N}(\boldsymbol{\mu})=\sum_{q=1}^{Q_{\mathcal{A}}} \Theta_{q}^{\mathcal{A}}(\boldsymbol{\mu}) \mathbb{A}_{N}^{q}, \quad \mathbb{F}_{N}(\boldsymbol{\mu})=\sum_{q=1}^{Q_{\mathcal{F}}} \Theta_{q}^{\mathcal{F}}(\boldsymbol{\mu}) \mathbb{F}_{N}^{q}, \quad \mathbb{Q}_{N}(\boldsymbol{\mu})=\sum_{q=1}^{Q_{\mathcal{Q}}} \Theta_{q}^{\mathcal{Q}}(\boldsymbol{\mu}) \mathbb{Q}_{N}^{q}
$$

being

$$
\left(\mathbb{A}_{N}^{q}\right)_{l m}=\mathcal{A}^{q}\left(\phi_{m}, \phi_{l}\right), \quad\left(\mathbb{F}_{N}^{q}\right)_{l m}=\mathcal{F}^{q}\left(\phi_{l}\right), \quad\left(\mathbb{Q}_{N}^{q}\right)_{l m}=\mathcal{Q}^{q}\left(\phi_{m}, \phi_{l}\right),
$$

so that all $\boldsymbol{\mu}$-independent algebraic structures can be computed only once and stored during the Offline stage. Moreover, expressing each basis $\phi_{i}$ of the space $X_{N}$ as an element of the FE space, $\phi_{l}(\boldsymbol{x})=\sum_{i=1}^{\mathcal{N}_{h}} \phi_{i}^{l} \boldsymbol{\xi}_{i}(\boldsymbol{x})$, where $\left\{\boldsymbol{\xi}_{i}\right\}_{i=1}^{\mathcal{N}_{h}}$ is an orthonormal basis of the space $X_{\mathcal{N}_{h}}$, we can denote $\mathbb{Z}_{N} \in \mathbb{R}^{\mathcal{N}_{h} \times N}$ the space matrix given by $\left(\mathbb{Z}_{N}\right)_{i l}=\phi_{i}^{l}$, with $1 \leq i \leq \mathcal{N}_{h}$, $1 \leq l \leq N$. It can be simply shown that this matrix allows to express the RB algebraic structures in terms of the analogous FE structures, by acting as a projection onto $X_{N}$ :

$$
\begin{array}{clc}
\mathbb{A}_{N}^{q}=\mathbb{Z}_{N}^{T} \mathbb{A}_{\mathcal{N}}^{q} \mathbb{Z}_{N}, & 1 \leq q \leq Q_{\mathcal{A}}, & \left(\mathbb{A}_{\mathcal{N}}^{q}\right)_{i j}=\mathcal{A}^{q}\left(\boldsymbol{\xi}_{j}, \boldsymbol{\xi}_{i}\right), \\
\mathbb{F}_{N}^{q}=\mathbb{Z}_{N}^{T} \mathbb{F}_{\mathcal{N}}^{q}, & 1 \leq q \leq Q_{\mathcal{F}}, & \left(\mathbb{F}_{\mathcal{N}}^{q}\right)_{i}=\mathcal{F}^{q}\left(\boldsymbol{\xi}_{i}\right), \\
\mathbb{Q}_{N}^{q}=\mathbb{Z}_{N}^{T} \mathbb{Q}_{\mathcal{N}}^{q} \mathbb{Z}_{N}, & 1 \leq q \leq Q_{\mathcal{Q}}, & \left(\mathbb{Q}_{\mathcal{N}}^{q}\right)_{i j}=\mathcal{Q}^{q}\left(\boldsymbol{\xi}_{j}, \boldsymbol{\xi}_{i}\right),
\end{array}
$$

Hence, during the Offline stage, all the structures (27) required by the RB machinery can be simply assembled and stored starting from the corresponding FE structures. In this way, the solution of the RB problem (24) can be efficiently performed online, for each value of the parameters $\boldsymbol{\mu} \in \mathcal{D}$, implying only handling (very) small algebraic structures. 
4. A posteriori error estimation. The Galerkin-type approximation allows to build an a posteriori residual-based error estimator w.r.t. the FE truth solution in order to certificate the RB approximation of the state solution [59, 49]. In the same way, a posteriori error bounds for linear and quadratic functionals of the state variables can also be derived. In general ${ }^{\mathrm{e}}$, denoting $r(W ; \boldsymbol{\mu})=\mathcal{F}(W ; \boldsymbol{\mu})-\mathcal{A}\left(U_{N}(\boldsymbol{\mu}), W, \boldsymbol{\mu}\right)$ the residual of the state equation and its dual norm

$$
\|r(\cdot ; \boldsymbol{\mu})\|_{X^{\prime}}=\sup _{W \in X} \frac{r(W ; \boldsymbol{\mu})}{\|W\|_{X}}
$$

we have that the following a posteriori error estimation is satisfied:

$$
\left\|U_{h}(\boldsymbol{\mu})-U_{N}(\boldsymbol{\mu})\right\|_{X} \leq \Delta_{N}(\boldsymbol{\mu}):=\frac{\|r(\cdot ; \boldsymbol{\mu})\|_{X^{\prime}}}{\beta_{h}^{L B}(\boldsymbol{\mu})},
$$

where $\beta_{h}^{L B}(\boldsymbol{\mu})$ is the lower bound of the discrete (parametric) inf-sup constant $\beta(\boldsymbol{\mu})$ defined in (22), computable by a linear programming algorithm (the successive constraint method, SCM, $[28,15])$. We remark that also the computations of the dual norm of the residuals, as well as of the lower bounds for the inf-sup constants, can be decoupled following the previous Offline-Online stratagem, in order to get an inexpensive evaluation of the error bound for each $\boldsymbol{\mu} \in \mathcal{D}$. More details can be found e.g. in [53], in [58] for the Stokes case, in [14, 69, 52, 43] for the steady Navier-Stokes case and in [32] for the unsteady Boussinesq equations. The same ingredients can be used to derive error bounds for linear and quadratic functionals, and certify also the solution of optimization, control and inverse problems [42]. More details on the way the certification of the forward problem impacts on the reliability of the inverse problem as well are reported in Sec. 7.

\section{EXAMPLE: STRUCTURAL PARAMETERS IDENTIFICATION IN A STENOTIC ARTERY}

Since arteries undergo large displacements that cannot be neglected when modelling blood flows, estimating their material parameters $[7,50]$ is crucial for accurate haemodynamical simulations. We present in this section a model problem related to the inverse identification of material parameters in a steady FSI problem, which can be of interest for predicting flows over stenosed portions of an artery.

\subsection{Formulation}

Following the approach presented in [36, 37], we consider the model reduction of an FSI model predicting flow over a simplified stenosis geometry of an artery. Here the fluid domain is chosen as $\Omega_{o}:=(0, L) \times\left(w_{s}, H+\eta\right)$, where $H$ and $L$ are the width and length of the artery in a reference configuration respectively, $\eta$ is the vertical displacement of the upper wall of the artery from the reference configuration, and $w_{s}$ is an idealised bottom wall shape function

$$
w_{s}\left(x_{1}\right):=\left\{\begin{aligned}
h \cos \frac{\pi\left(x_{1}-x_{s}\right)}{2 \delta} & \text { if } x_{s}-\delta<x_{1}<x_{s}+\delta \\
0 & \text { otherwise, }
\end{aligned}\right.
$$

where $\delta>0$ is the width, $x_{s}$ the centerpoint, and $h$ the height of the stenosis. In the following, we denote by $\boldsymbol{u}=\left(u_{x}, u_{y}\right)$ and $p$ the velocity and the pressure of the flow, respectively.

In order to close the system we need to provide an equation for solving the displacement $\eta$ (as mentioned when introducing (8)). The equilibrium equation for the structural displacement is chosen

\footnotetext{
${ }^{e}$ For the sake of simplicity we report here a very general expression for the error bound in the case of a generalized noncoercive problem (e.g. Stokes problem).
} 
as the following second order equation with a fourth order perturbation (with $\varepsilon>0$ small)

$$
\varepsilon \frac{\partial^{4} \eta}{\partial x_{1}^{4}}-k G h \frac{\partial^{2} \eta}{\partial x_{1}^{2}}+\frac{E h}{1-\nu_{P}^{2}} \frac{\eta}{R_{0}\left(x_{1}\right)^{2}}=\tau_{\Gamma_{w}}, \quad x_{1} \in(0, L),
$$

where $h$ is the wall thickness, $k$ is the Timoshenko shear correction factor, $G$ the shear modulus, $E$ the Young modulus, $\nu_{P}$ the Poisson ratio, $R_{0}$ the radius of the reference configuration, and $\tau_{\Gamma_{w}}$ denotes the traction applied to the wall by the fluid inside the domain $\Omega_{0}$ [54]. We have added a fourth order singular perturbation term (in order to model biological tissues we should have $\varepsilon \ll 1$ ) in order to ensure sufficient regularity for the displacement and to allow convergence of the partitioned solution algorithm. The weak form of (30) is to find the structural displacement in the normal direction $\eta \in D$ s.t.

$$
\mathcal{E}(\eta, \phi)=\tau_{\Gamma_{w}}(\phi), \quad \forall \phi \in D
$$

being

$$
\mathcal{E}(\eta, \phi)=\varepsilon \int_{0}^{L} \frac{\partial^{2} \eta}{\partial x_{1}^{2}} \frac{\partial^{2} \phi}{\partial x_{1}^{2}} d x_{1}+k G h \int_{0}^{L} \frac{\partial \eta}{\partial x_{1}} \frac{\partial \phi}{\partial x_{1}} d x_{1}+\frac{E h}{1-\nu_{P}^{2}} \int_{0}^{L} \frac{\eta \phi}{R_{0}\left(x_{1}\right)^{2}} d x_{1}
$$

and $D:=H_{0}^{2}(0, L)$ the space of kinematically admissible displacements.

\subsection{Coupling of fluid and structure}

The fluid and structure are coupled together by taking the applied traction $\tau_{\Gamma_{w}}$ to be the normal component of the normal Cauchy stress of the fluid on $\Gamma_{w}$, i.e.

$$
\tau_{\Gamma_{w}}=(\boldsymbol{\sigma}(\boldsymbol{u}, p) \boldsymbol{n}) \cdot \boldsymbol{n}, \quad \text { on } \Gamma_{w} .
$$

This can be expressed in the weak sense using the residual $\mathcal{R}(\cdot ; \boldsymbol{u}, p ; \boldsymbol{\mu}) \in\left(V_{h}^{w}\right)^{\prime}$ of the fluid solution on the interface defined, for all $\boldsymbol{w} \in \mathcal{V}_{h}^{w}$, as (see the Appendix A for the definition of the operators related to the fluid equations)

$$
\mathcal{R}(\boldsymbol{w} ; \boldsymbol{u}, p ; \boldsymbol{\mu}):=\left\langle F^{s}(\boldsymbol{\mu}), \boldsymbol{w}\right\rangle-a(\boldsymbol{u}+\boldsymbol{g}, \boldsymbol{w} ; \boldsymbol{\mu})-b(p, \boldsymbol{w} ; \boldsymbol{\mu})-c(\boldsymbol{u}+\boldsymbol{g}, \boldsymbol{u}+\boldsymbol{g}, \boldsymbol{w} ; \boldsymbol{\mu}),
$$

where $V_{h}^{w} \supset V_{h}$ is the same finite-element space for the velocity except that the essential boundary condition is not imposed on the wall $\Gamma_{w}$. Otherwise said, $\boldsymbol{w}$ functions are free to behave at finite element nodes on $\Gamma_{w}$. In [36] we solved the nonlinear coupled problem ((32-45)) with a parametric coupling method. A partitioned algorithm was presented for coupling together the fluid and structure by parametrizing the computational domain (and consequently the displacement) for the fluid, and measuring the coupling error in the parametric displacement space. Here we apply the same method for solving the steady fluid-structure interaction problem.

Since the fluid domain is parametrized, we can apply the reduced basis method outlined before to reduce the computational cost by replacing the discrete Navier-Stokes equations with their RB counterparts. At each iteration $k$ of the algorithm we must then solve a least-squares problem:

$$
\min _{\boldsymbol{\pi}^{k+1} \in \mathcal{D}} \int_{\Gamma_{w}}\left\|\eta\left(\boldsymbol{\pi}^{k+1}\right)-\mathcal{S} \circ \mathcal{L} \circ \mathcal{F}\left(\boldsymbol{\pi}^{k}\right)\right\|^{2} d \Gamma=: \min _{\boldsymbol{\pi}^{k+1} \in \mathcal{D}} J(\boldsymbol{\mu})
$$

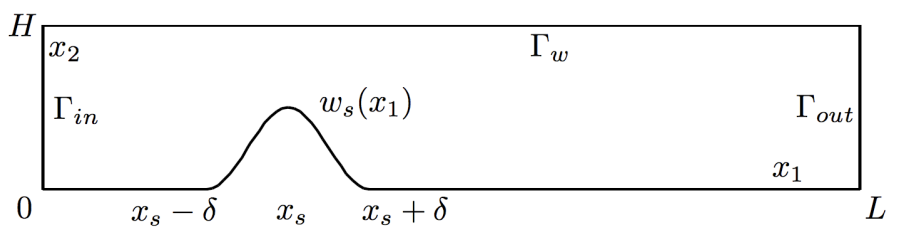

Figure 2. Definition of the geometrical domain for an idealised stenotic artery case. 
to find the configuration of the fluid domain $\Omega_{o}\left(\pi^{k+1}\right)$ at the next iteration, where $\mathcal{F}\left(\pi^{n}\right)$ represents the resolvant operator giving the fluid solution in the domain $\Omega_{o}\left(\boldsymbol{\pi}^{n}\right), \mathcal{L}(\cdot)$ is an operator transferring the loads from fluid to structure in a weak way using the fluid residual $(33), \mathcal{S}(\cdot)$ represents the resolvant operator giving the structural displacement assuming the applied loads, and $\eta(\boldsymbol{\pi})$ is the parametric displacement of the fluid domain at configuration $\Omega_{o}(\boldsymbol{\pi})$.

\subsection{Numerical results}

We consider the computational reference domain $\Omega=(0, L) \times\left(w_{s}, 1\right)$, with $L=5$, and the corresponding computational mesh is represented in Fig. 3. Lenghts are expressed in centimeters (cm). For the FE discretization we use $\mathbb{P}_{2} / \mathbb{P}_{1}$-elements - this choice gives a total of $\mathcal{N}_{h} \approx 35,000$ degrees of freedom for the finite element "truth" approximation. The viscosity is chosen as the physiological value $\nu=0.035 \mathrm{~g} / \mathrm{cm} \cdot \mathrm{s}$, and the parabolic inflow velocity fixed as $\boldsymbol{g}(y)=(5(1-$ $\left.\left.(y-0.5)^{2} / 0.25\right), 0\right) \mathrm{cm} / \mathrm{s}$. We consider steady incompressible Navier-Stokes flow with a Reynolds number of about $R e=80$ at the location of the stenosis.

The parametrization of the fluid domain displacement is performed with a $12 \times 2$ regular grid of control points, where only the 8 central points on the upper row were allowed to move freely in the $y$ direction. The two left- and rightmost columns of control points are kept fixed in order to guarantee a parametric displacement that conforms to the boundary conditions set upon the structure.

Using a stopping tolerance of $\varepsilon_{\text {tol }}^{E I M}=10^{-4}$ in the $L^{\infty}$-norm for the EIM, the total number of affine terms is $Q=106$. The admissible parameter range for the displacement of each FFD control point (see Fig. 3) is $\mu_{p} \in[-0.25,0.25]$ for $p=1,2, \ldots, 8$. The RB space is built by means of the greedy algorithm using a stopping tolerance of $\varepsilon_{t o l}^{R B}=5 \times 10^{-2}$ and is made by $N=8$ basis functions.

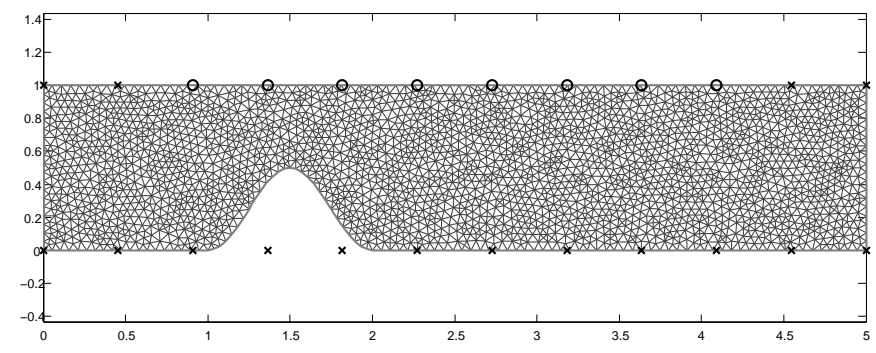

Figure 3. Reference domain $\Omega$, "truth" finite element mesh, and FFD control points used to model the displacements of the upper wall for the FSI problem. Only eight control points are allowed to move.

In Fig. 4 we display a RB simulation of the flow field, namely the velocity streamlines in the case that the artery is rigid and does not deform. The stenosis induces a strong double vortex downstream, resulting in an area of low wall shear stress (WSS) immediately after the stenosed part. The presence of the vortices in the reduced solution already with quite few basis functions highlights the fact that important physical features of the flow can be captured by the reduced basis method as long as the features are part of the snapshots used to construct the basis (i.e. recirculation zones, viscous boundary layers, etc.). Thus a proper sampling procedure via the greedy algorithm is absolutely mandatory to guarantee a good selection of states for the reduced system.

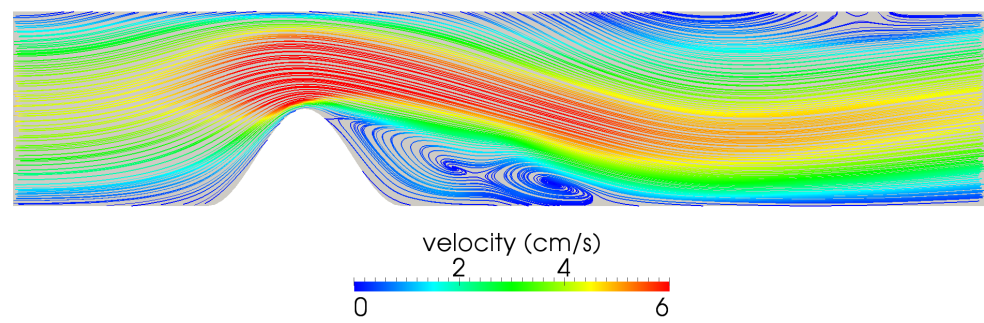

Figure 4. Velocity magnitude and streamlines of the flow in a rigid stenosed artery (RB simulation). 

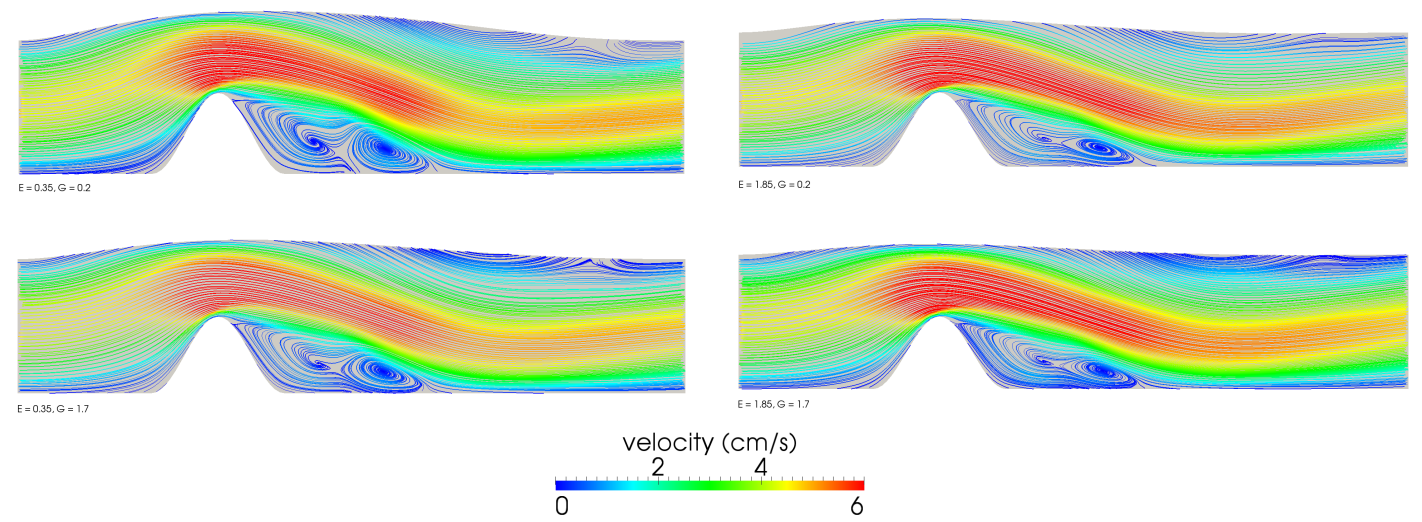

Figure 5. Velocity magnitude and streamlines of the steady incompressible Navier-Stokes flow in compliant stenosed artery (RB simulation) for different elastic moduli values, $E \in\{0.35,1.85\}$ and $G \in\{0.2,1.7\}$.

The shape of the upper wall has a very strong effect on the type of vortices created and ultimately the potential growth of the stenosis. To explore the uncertainty related to the arterial wall properties we define the uncertainty parameters $\omega=(G, E)$ as the shear modulus and Young modulus, where the ranges considered ${ }^{\mathrm{f}}$ were $G \in[0.2,1.7] \cdot 10^{6} \mathrm{dyn} / \mathrm{cm}^{2}$ and $E \in[0.35,1.85] \cdot 10^{6} \mathrm{dyn} / \mathrm{cm}^{2}$. The other model parameters were chosen as $\nu_{P}=0.5, K=0.9643$, and $h=0.1 \mathrm{~cm}$. The fourth-order perturbation term was chosen as $\varepsilon=0.1$ to obtain reasonable displacements and convergence for the partitioned algorithm (see e.g. [19] for details).

The mechanobiological response of the tissue to flow disturbances is a complex phenomena to model mathematically. Clinical evidence indicates that increased plaque growth is observed in regions of abnormally low or oscillating WSS [31, 33]. In conjuction the tissue undergoes a maladaptive process, resulting in a marked change in the mechanical properties of the arterial wall. Several different derived functions of WSS have been proposed for quantifying and predicting the likely plaque buildup locations. Many of them rely on the oscillatory shear index, which our simplified model is unable to capture. If one is only interested in estimating the mean WSS distribution, the unsteady and pulsatile flow can be replaced with a steady mean flow with the same Reynolds number [17, 41].

As a simplification we look at four different output functionals: the total viscous energy dissipation

$$
J_{1}(\boldsymbol{\mu})=\frac{\nu}{2} \int_{\Omega_{o}}|\nabla \boldsymbol{u}|^{2} d \Omega_{o}
$$

the minimum downstream WSS (being $\Gamma_{\text {wall }}=\left(x_{s}+\delta, L\right)$ )

$$
J_{2}(\boldsymbol{\mu})=\nu \min _{x \in \Gamma_{\text {wall }}}\left\{\left.\frac{\partial u_{x}(x, y)}{\partial y}\right|_{y=0}\right\}
$$

the mean downstream WSS

$$
J_{3}(\boldsymbol{\mu})=\frac{\nu}{\left|\Gamma_{\text {wall }}\right|} \int_{\Gamma_{\text {wall }}}\left[\left.\frac{\partial u_{x}(x, y)}{\partial y}\right|_{y=0}\right] d x,
$$

and the mean pressure drop in the stenosed section

$$
J_{4}(\boldsymbol{\mu})=\int_{\Gamma_{\text {in }}} p d y-\int_{\Gamma_{\text {out }}} p d y
$$

The first output measures the total energy loss in the flow due to the stenosed section, including recirculation effects, and the second and third outputs are local indicators of low WSS and possible vortices related to recirculation. In clinical practice many noninvasive measurement methods are aimed at quantifying atherosclerosis risk by identifying the distending pressure inside the artery [48], which we simulate in this simplified example by the fourth output.

\footnotetext{
${ }^{f}$ The unit $10^{6} \mathrm{dyn} / \mathrm{cm}^{2}$ is omitted from hereon in for brevity and should be implicitly understood.
} 

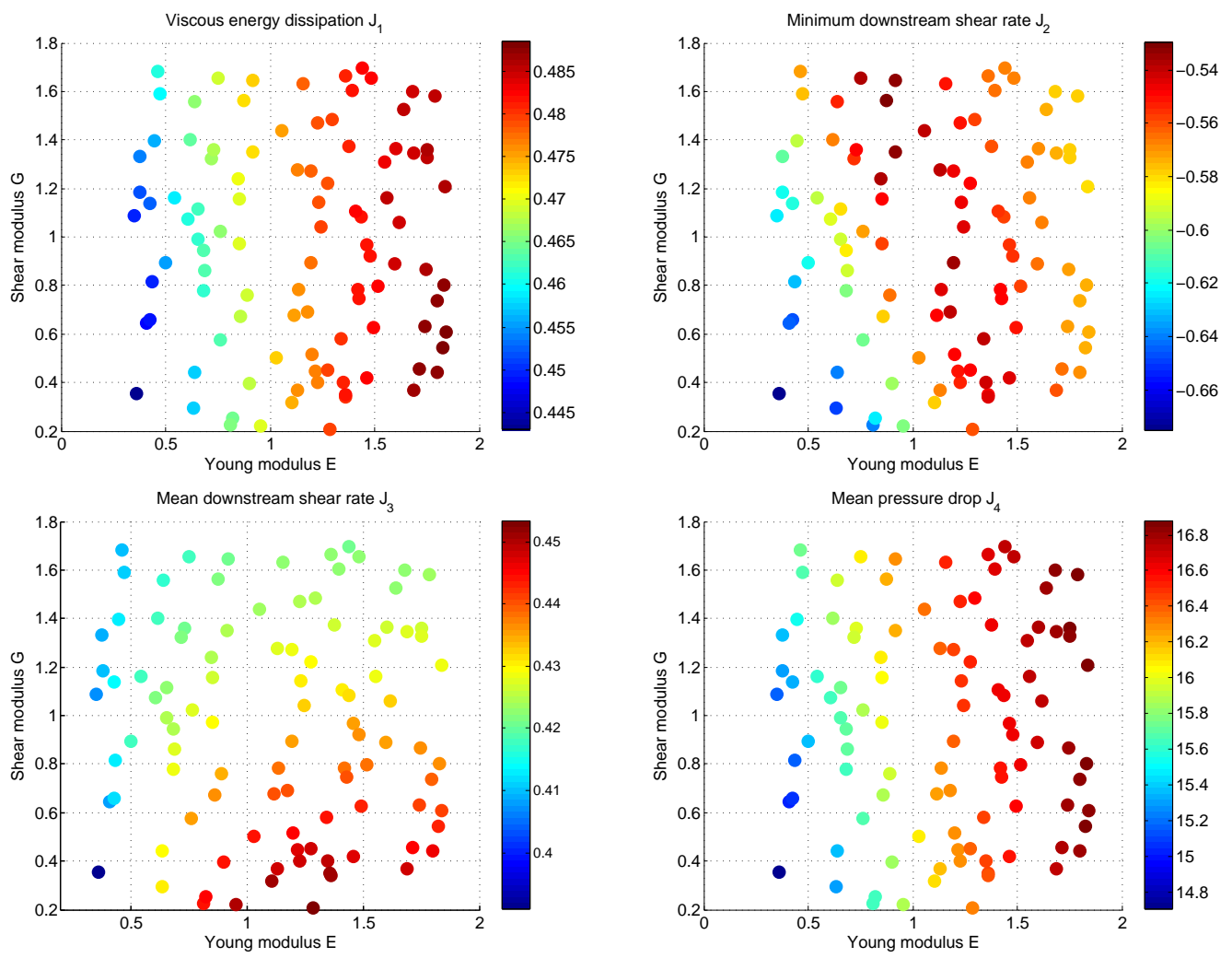

Figure 6. Observations of the flow in the compliant stenosed artery: values of the four output functionals $J_{1}$, $J_{2}, J_{3}$ and $J_{4}$ at randomly sampled points in the parametric uncertainty range (RB simulations).

In Fig. 5 the RB simulation of the flow is given for each of the four corners of the parametric uncertainty domain. It can be observed that the more compliant the arterial wall is, the larger is the recirculation region created behind the stenosis. In Fig. 6 we present the output functionals $J_{1}, J_{2}$, $J_{3}$, and $J_{4}$ evaluated at 100 randomly selected sample points in the parametric uncertainty region.

Some conclusions can be drawn:

1. The effect of the Young modulus $E$ is considerably larger than the effect of the shear modulus $G$ on both functionals (35)-(36), at least for this simplified structural model.

2. The viscous dissipation is a monotonic function of the Young modulus, i.e. the stiffer the arterial wall the more dissipation is observed. Increased arterial stiffness has been linked to increased risk of atherosclerosis $[68,71]$, so that the viscous dissipation is a possible indicator for atherosclerosis risk.

3. The minimum WSS depends primarily on the Young modulus and behaves in a nonlinear way. There seems to be an optimal range of the Young modulus $(E \geq 0.7)$, after which the observed WSS starts to go down again. This is likely due to the fact that further increasing of the compliance of the structure leads to a larger recirculation zone and a strong separation of the flow from the bottom wall.

4. The mean downstream WSS has different behavior from the minimum downstream WSS. This is likely due to the strong local WSS induced by the jet impacting the downstream wall.

\subsection{Inverse problem of determining elastic moduli based on the measured pressure drop}

As mentioned before, increased stiffness of the arterial wall is linked with increased risk of atherosclerosis. Within this context, one of the most important inverse problems of haemodynamics [55] is concerned with the possibility of identifying the material properties of the arterial wall in a segment of an artery upon measuring flow rate and pressure over the segment. 
Several approaches exploit e.g. ultra-sound Doppler measurements of the simultaneous flow variations and wall displacements, so that viscoelastic parameters are estimated by fitting the theoretical constitutive equations to the experimental measurements (see e.g. [4, 5, 48]). Here we consider a simplified version of this problem: assuming the stenosis geometry described above, if the observation $s^{*}:=J_{4}\left(\boldsymbol{\mu}^{*}\right)$ (the mean pressure drop) is measured is it possible to determine the Young modulus $E$ and the shear modulus $G$ ?

From the Fig. 6 it is clear that in general the shear modulus can not be reliably identified, so we concentrate first on identification of the Young modulus, assuming that the value of the shear modulus is known. We demonstrate the solution of the deterministic inverse problem for two different observed values of the Young modulus: $E=1.2334 \cdot 10^{6} \mathrm{dyn} / \mathrm{cm}^{2}$ and $E=0.9175 \cdot 10^{6}$ $\mathrm{dyn} / \mathrm{cm}^{2}$, and a fixed value $G=0.5000 \mathrm{dyn} / \mathrm{cm}^{2}$ for the shear modulus. The corresponding values for the pressure drops were $J_{4}=16.3881 \mathrm{dyn} / \mathrm{cm}^{2}$ and $J_{4}=15.9390 \mathrm{dyn} / \mathrm{cm}^{2}$. In the first case we assume $1 \%$ additive noise in the measurements, in the second case $3 \%$ noise. The results of the inverse identification problem without regularization are given in Table I for one particular noise realization. For the first case a very good reconstruction of the value of the Young modulus is obtained, while in the second case the estimate is quite poor. This is due to the low sensitivity of the pressure drop $J_{4}$ to the material parameters, leading to increasingly poor reconstruction as the noise level is increased.

Table I. Results of the deterministic inverse problem of haemodynamics in two different cases

\begin{tabular}{ccccc}
\hline Young modulus $E$ & Inverse estimate & Relative error & \# of optim iters & \# of PDE solves \\
\hline 1.2334 & 1.2253 & $0.66 \%$ & 10 & 45 \\
0.9175 & 0.6501 & $29.1 \%$ & 9 & 41 \\
\hline
\end{tabular}

Following instead the Bayesian approach, we provide a probability distribution function for the elastic moduli $(E, G)$ encapsulating the uncertainties related to measurements. If we assume that the prior distribution is $p_{E, G}(E, G) \sim \mathcal{U}([0.35,1.85],[0.2,1.7])$, i.e. $E$ and $G$ are two independent uniformly distributed random variables, and that the measurements of the pressure drop are independent and normally distributed variables with expected value equal to the true mean pressure drop and variance $\sigma^{2}$, i.e. $p_{s^{*} \mid E, G}\left(s^{*} \mid E, G\right)=p_{\text {noise }}\left(s^{*}-J_{4}(E, G)\right) \sim \mathcal{N}\left(0, \sigma^{2}\right)$, then we obtain the posterior probability density $p_{E, G \mid s^{*}}\left(E, G \mid s^{*}\right)$ given by

$$
p_{E, G \mid s^{*}}\left(E, G \mid s^{*}\right) \propto\left\{\begin{array}{cl}
\exp \left[-\frac{\left(s^{*}-J_{4}(E, G)\right)^{2}}{2 \sigma^{2}}\right], & \text { if }(E, G) \in[0.35,1.85] \times[0.2,1.7] \\
0, & \text { otherwise. }
\end{array}\right.
$$

The posterior distribution $p_{E, G \mid s^{*}}\left(E, G \mid s^{*}\right)$ for the two different test cases and their observations $s^{*}$ are shown in Fig. 7. In both cases we see that only the Young modulus $E$ is identifiable from the
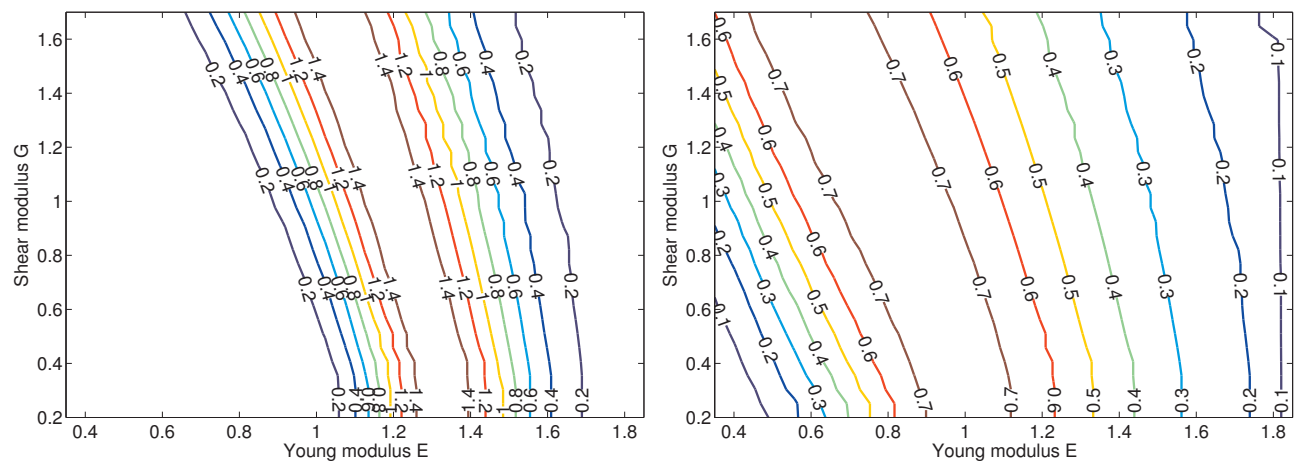

Figure 7. Contour plot of the posterior distribution $p_{E, G \mid s^{*}}\left(E, G \mid s^{*}\right)$ for two different cases: $\left(s^{*}, \sigma\right)=$ $(16.3881,0.17)$ and $\left(s^{*}, \sigma\right)=(15.9390,0.51)$. Only the Young modulus $E$ can be reliably identified. 
pressure drop alone, and the value of the shear modulus $G$ can have an effect on the maximum likelihood estimate of $E$. From the second case we can observe that increasing the noise very quickly smears out the probability distribution. Even when the arterial configuration is known, the inverse problem of haemodynamics is indeed ill-conditioned and susceptible to noise; further difficulties arise when the geometrical configuration is recovered e.g. from medical images. A purely deterministic approach to the solution of this inverse problem can therefore mask the underlying uncertainty and lead us to erroneously optimistic conclusions about the quality of inverse estimates such as those dictated in Table I. If no prior information is at hand concerning the indeterminable parameters, such as the shear modulus, the inverse problem of haemodynamics can not be reliably solved.

\subsection{Inverse problem of determining downstream WSS based on the measured pressure drop}

Alternatively we can pose the question: if we have a measurement of the pressure drop, $s^{*}=J_{4}\left(\boldsymbol{\mu}^{*}\right)$, can we predict the WSS on the arterial wall downstream from the stenosis? Even if we have shown that the elastic moduli of the arterial wall are not strictly identifiable from the pressure drop measurement alone, it might still be possible that the WSS (which in the case of predicting stenosis risk is the primary quantity of interest) can be reasonably accurately identified. In this way the pressure drop measurement would act as a surrogate for identifying the WSS.

As the value of the mean WSS $J_{3}(\boldsymbol{\mu})$ can be obtained by performing the forward simulation given the material parameters $\boldsymbol{\omega}$, we can explore the posterior p.d.f. $p_{J_{3} \mid s^{*}}\left(J_{3}(\boldsymbol{\mu}) \mid s^{*}\right)$ provided by means of the Metropolis-Hastings algorithm, introduced in Sec. 2. In Fig. 8 we display the observed histograms for the distribution of the minimum WSS $J_{2}$ and the mean WSS $J_{3}$ for the case $\left(s^{*}, \sigma\right)=(16.3881,0.17)$. The range of the plot is scaled to correspond with the entire range of observed variation in each output. Thus the pressure drop can indeed be used to determine the minimum value of the WSS downstream from the stenosis, while the mean WSS distribution is more smeared out. This is due to the fact that the unidentifiable shear modulus $G$ has a larger effect on the mean WSS than the minimum WSS. In Fig. 9 we display the observed histograms for the distribution of the minimum WSS $J_{2}$ and the mean WSS $J_{3}$ for the case $\left(s^{*}, \sigma\right)=(15.9390,0.51)$.
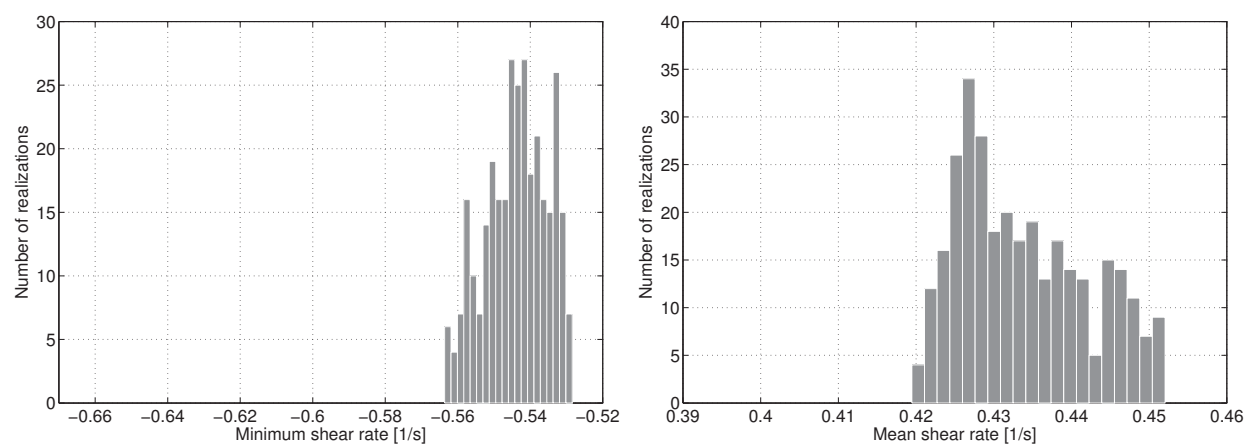

Figure 8. Histograms of posterior distributions $p_{J_{2} \mid s^{*}}\left(J_{2}(E, G) \mid s^{*}\right)$ (left) and $p_{J_{3} \mid s^{*}}\left(J_{3}(E, G) \mid s^{*}\right)$ (right) for $\left(s^{*}, \sigma\right)=(16.3881,0.17)$; the distributions were sampled using the Metropolis-Hastings algorithm.

The exploration of the parameter space with 100 samples to produce the Fig. 6 took around 70 minutes of computational time ${ }^{g}$ when all the fluid simulations were performed with the use of the reduced basis method. For the sake of comparison, we estimate that exploring the same parameter space with the full finite element simulation would have taken around 33 hours of computational time - and considerably more if the fluid-structure interaction problem had been solved using standard methods and not taking advantage, as done here, of the geometrical reduction afforded by the parametrization of the structural deformation.

${ }^{g}$ Computations have been executed on a personal computer with $2 \times 2 \mathrm{GHz}$ Dual Core AMD Opteron (tm) processors $2214 \mathrm{HE}$ and $16 \mathrm{~GB}$ of RAM. 

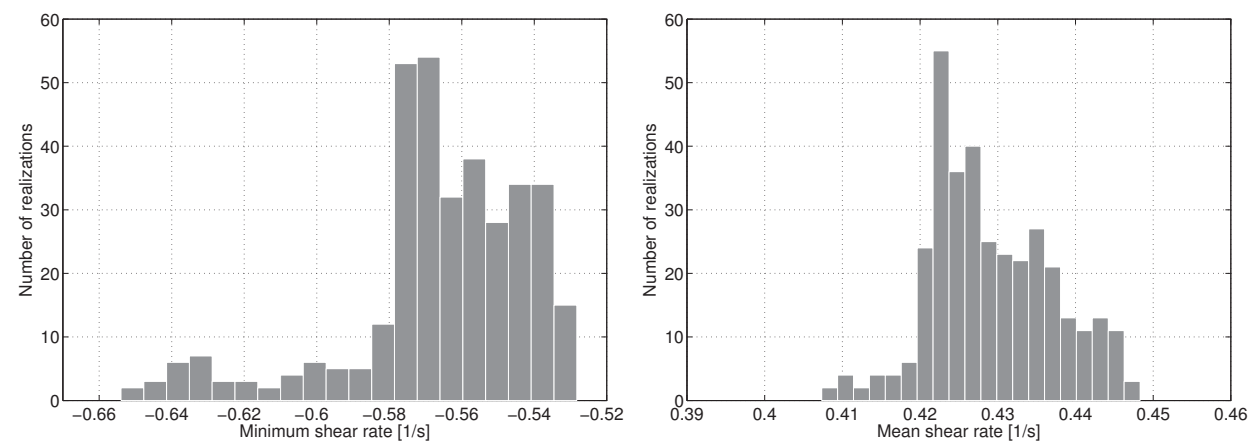

Figure 9. Histograms of posterior distributions $p_{J_{2} \mid s^{*}}\left(J_{2}(E, G) \mid s^{*}\right)$ (left) and $p_{J_{3} \mid s^{*}}\left(J_{3}(E, G) \mid s^{*}\right)$ (right) for $\left(s^{*}, \sigma\right)=(15.9390,0.51)$; the distributions were sampled using the Metropolis-Hastings algorithm.

\section{EXAMPLE: ROBUST SHAPE DESIGN OF FEMORO-POPLITEAL BYPASS GRAFTS}

Several surgical procedures can be applied in order to restore the normal blood supply to the downstream vessels, in case of severely stenosed arteries, caused by the accumulation of fatty materials (atherosclerosis). Among these, we mention angioplasty and grafting. In the first case, a catheter is advanced from a peripheral artery to the site of stenosis, which is then disrupted by dilating it with a balloon used to expand a wire mesh stent which supports the vessel walls. In the second case, we can either remove the diseased artery and replace it with another vessel sutured end-to-end with the remaining arteries (vessel replacement), or provide an alternative bridging path (or bypass) in order to overcome the stenoses, which are left in place.

Bypass grafts are used in both coronary artery disease to recover the blood perfusion of the cardiac muscle and peripheral vascular disease, such as femoro-poplitheal grafts, which are used to bypass blockages in the femoral artery. Flow separation, recirculation, and stagnation close to the anastomosis region is strongly dependent on both the local artery geometry and the grafting shape, and linked to plaque formation, which may lead to vessel narrowing (occlusions) or intimal thickening (stenosis). As a consequence of narrowing and intimal thickening, the bypass may undergo a severe reocclusion and thus a complete failure some years after surgery.

\subsection{Formulation}

In this framework, shape optimization techniques [23] may in principle allow the design of better bypass grafts in order to minimize the risk of restenosis, or even developing new blockages. We consider the optimal shape design of a femoro-poplitheal bypass, and model the fluid by the incompressible steady Navier-Stokes model, as described in Appendix A. The fluid domain is parametrized by means of a FFD mapping $T(\cdot ; \boldsymbol{\pi})$, discussed in the following subsection. In particular, we assume to deal with a partially blocked host artery, whose degree of occlusion is uncertain and described by a parametrized Dirichlet condition $\phi_{\text {res }}(\omega) \mathbf{g}_{\text {res }}(\mathbf{x})$ depending on an uncertain parameter $\omega \in \mathcal{D}_{\omega}$.

Several options for the cost functional will be discussed, both to obtain an optimized graft shape and to measure the effect of the uncertainty in the residual flow. The most common choices in view of reduction or elimination of spiraling streamlines are based on the observation of the vorticity in the subregion $\Omega_{o}^{o b s}$ within which vortices suppression is desired (see Fig. 10):

$$
J_{1}(\boldsymbol{\mu})=\int_{\Omega_{o}^{o b s}}|\nabla \times \boldsymbol{u}|^{2} d \Omega_{o}
$$

or a tracking-type cost functional like

$$
J_{2}(\boldsymbol{\mu})=\int_{\Omega_{o}^{o b s}}\left|\boldsymbol{u}-\boldsymbol{u}_{\text {stokes }}\right|^{2} d \Omega_{o},
$$

being $\boldsymbol{u}_{\text {stokes }}$ the solution of the Stokes problem with the same data. 


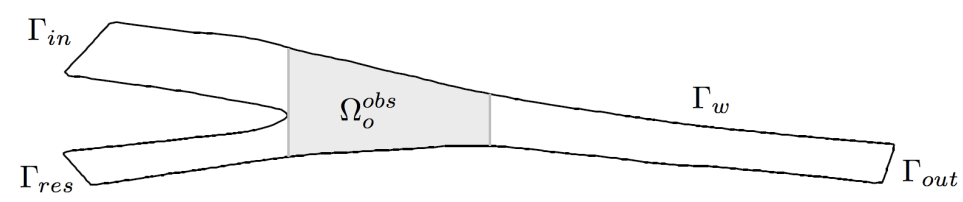

Figure 10. Definition of the subregion $\Omega_{o}^{o b s}$ used to measure the appearance of a vortex core.

Clearly, we are interested in the minimization of the vorticity in the downfield subregion, where occlusions and plaque formation usually occur; nevertheless, lower vorticities may be obtained not only by reducing vortices, but also by widening the graft and decreasing wall shear stresses, which have a strong impact on vorticity layers near the walls. In order to take into account the former contribution, we shall focus on the region $\Omega_{o}^{o b s}$ where a vortex may occur (see Fig. 10).

Vortex cores are related to regions where the eigenvalues of $\nabla \boldsymbol{u}$ are complex - in the twodimensional case this is equivalent to $\operatorname{det}(\nabla \boldsymbol{u})>0$. Thus a possible choice for vortex identification is given by the following Galilean invariant functional:

$$
\tilde{J}_{3}(\boldsymbol{\mu})=\int_{\Omega_{o}^{\text {obs }}} \max (0, \operatorname{det} \nabla \boldsymbol{u}) d \Omega_{o} .
$$

This cost functional has been proposed in [24, 34] for optimal control of nonstationary flows and used in [30] also for stationary viscous flows. An equivalent expression for $\tilde{J}_{3}(\boldsymbol{\mu})$ is given by

$$
J_{3}(\boldsymbol{\mu})=\int_{\Omega_{o}^{o b s}} h(\operatorname{det} \nabla \boldsymbol{u}) d \Omega_{o},
$$

where the smoothing function $h(t)$ defined by

$$
h(t)= \begin{cases}0 & t \leq 0 \\ t^{3} /\left(t^{2}+1\right) & t>0\end{cases}
$$

is introduced in order to avoid the nondifferentiable max-operation in $\tilde{J}_{3}(\boldsymbol{\mu})$. Moreover, we observe that in the case of $J_{2}(\boldsymbol{\mu})$ and $J_{3}(\boldsymbol{\mu})$ vorticity quantification is not obtained in terms of intrinsic properties of velocity and pressure fields. As for the previous application, since the fluid domain is parametrized, we can apply the RB method at each iteration of the optimization subroutine to reduce the cost of each input/output evaluation. In particular, we exploit an affine-scaling interiorpoint Newton method, where at each step we replace the discrete Navier-Stokes equations with their RB counterparts. Each evaluation is made on the reference domain, updating the parametrized tensors to consider the effect of geometrical changes.

\subsection{Numerical results}

We consider the computational reference domain represented in Fig. 11; for the FE discretization we introduce $\mathbb{P}_{2} / \mathbb{P}_{1}$-elements - this choice gives a total of $\mathcal{N}_{h} \approx 16,000$ degrees of freedom for the finite element "truth" approximation. The viscosity is chosen as the physiological value $\nu=0.035 \mathrm{~g} / \mathrm{cm} \cdot \mathrm{s}$, while the inflow velocities on $\Gamma_{i n}$ (bypass) and $\Gamma_{r e s}$ (blocked host artery) are given by a Poiseuille profile $\phi_{i n}(\omega) \mathbf{g}_{i n}(\mathbf{x})$ and a bell-shape profile with very small variance profile $\phi_{\text {res }}(\omega) \mathbf{g}_{\text {res }}(\mathbf{x})$, respectively. The first one represents a fully developed flow, while the second one models a residual flow in the blocked host artery. In particular, the dependence of the two flows from the uncertain parameter $\omega \in \mathcal{D}_{\omega}=[0,15]$ is such that the downfield flowrate is constant, with a flow split ranging from 1/0 (complete occlusion of the host artery, for $\omega=0$ ) to 2/1 (flowrate across the occluded artery equal to one half of the flowrate across the graft, for $\omega=15)$.

We consider steady incompressible Navier-Stokes flow with around $\operatorname{Re}=150$ at the location of the anastomosis. The parametrization of the shape deformation is performed with a $6 \times 4$ regular grid of control points, where only 6 points are allowed to move freely in the $y$-direction. 


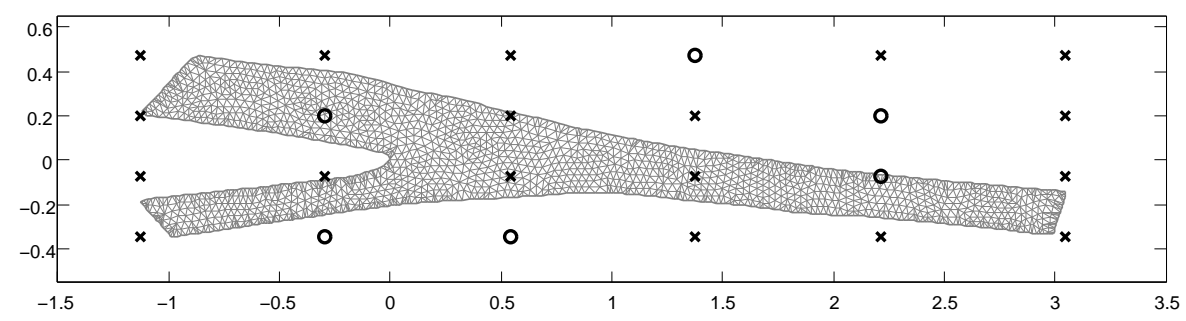

Figure 11. Reference domain $\Omega$, "truth" finite element mesh, and FFD control points used to model the displacements of the shape for the shape optimization problem. Only six control points are allowed to move.

In particular, the allowed control points (denoted in Fig. 11 with a cross $\times$ ) have been selected by means of a greedy procedure as those points which maximize the sensitivities of an energy functional with respect to their displacements. Using a stopping tolerance of $\varepsilon_{t o l}^{E I M}=10^{-4}$ in the $L^{\infty}$-norm for the EIM, the total number of affine terms is $Q=289$. The admissible parameter range is $\pi_{p} \in[-0.2,0.2]$ for $p=1,2, \ldots, 6$. A seventh parameter $\omega \in[0,15]$ takes into account the magnitude of the residual flow - and thus the flow splitting. The RB space, built by means of a greedy algorithm with a stopping tolerance $\varepsilon_{t o l}^{R B}=5 \times 10^{-3}$, is made by $N=20$ basis functions.

In Fig. 12 the results of the shape optimization problem for each cost functional $J_{i}(\boldsymbol{\mu}), i=1,2,3$ are reported, in case of fixed degrees of occlusion; indeed, $N_{\text {opt }}=16$ shape optimization problems have been solved, corresponding to the cases $\omega=0,1, \ldots, 15$. We can notice that the minimum values obtained with the vorticity functional $J_{1}(\boldsymbol{\mu})$ and the functional based on Galilean invariant $J_{3}(\boldsymbol{\mu})$ are decreasing function with respect to $\omega$, an indication that the case $\omega=0$ is the most difficult one concerning vorticity minimization.
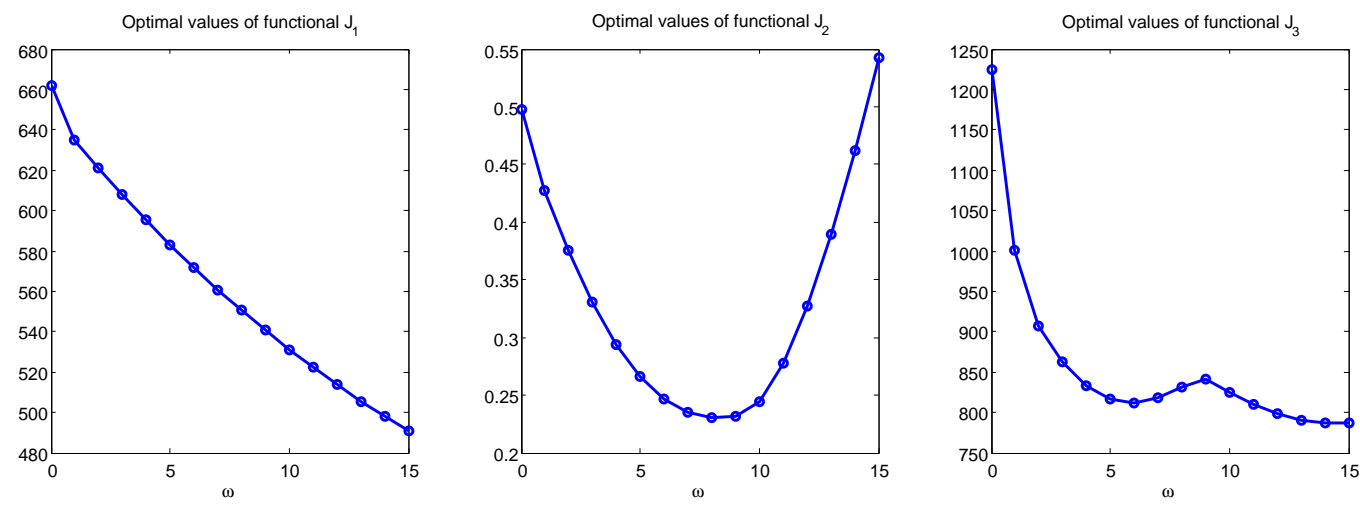

Figure 12. Minimum values for the three cost functionals as a function of the unknown residual flow parameter $\omega$. Results obtained by shape optimization for $N_{o p t}=16$ different cases between $\omega=0,1, \ldots, 15$.

As expected, the condition leading to the strongest development of vorticity cores is the presence of a complete occlusion, for which the flow through the bypass starts creating a strong vortex in the proximity of the anastomosis. As soon as a small residual flow crosses the occluded branch, the vortex cores are removed. On the other hand, also the tracking-type functional detects the presence of the vortex core for small values of $\omega$, but for larger values of $\omega$ the misfit between the two flows increases considerably due to the presence of the strong nonlinear convective term. Moreover, the reduction of the cost functional for the three cases ranges from $53 \%$ to $73 \%$, averaging the results on the $N_{\text {opt }}=16$ problems for each case (see Table II). In Fig. 13 the velocity fields for the initial and the optimal shapes obtained with the three cost functionals are reported, considering the minimum $(\omega=0)$ and the maximum $(\omega=15)$ magnitude of the residual flow across the occluded branch. The vorticity cores are clearly observable in presence of a complete artery blockage; moreover, although we get a sensible reduction of the cost functional also in this case, the vorticity cores never disappear completely. 

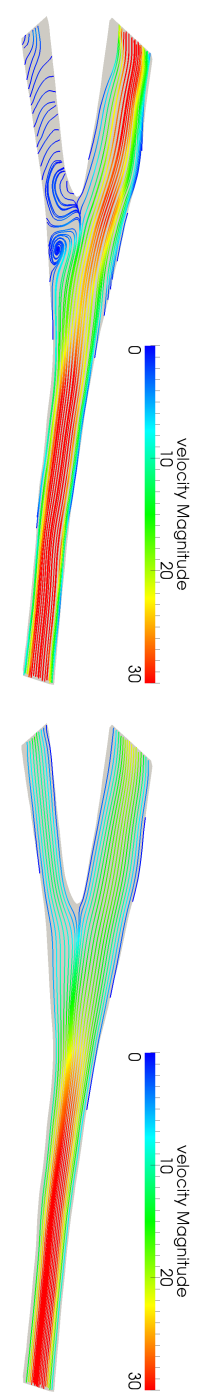
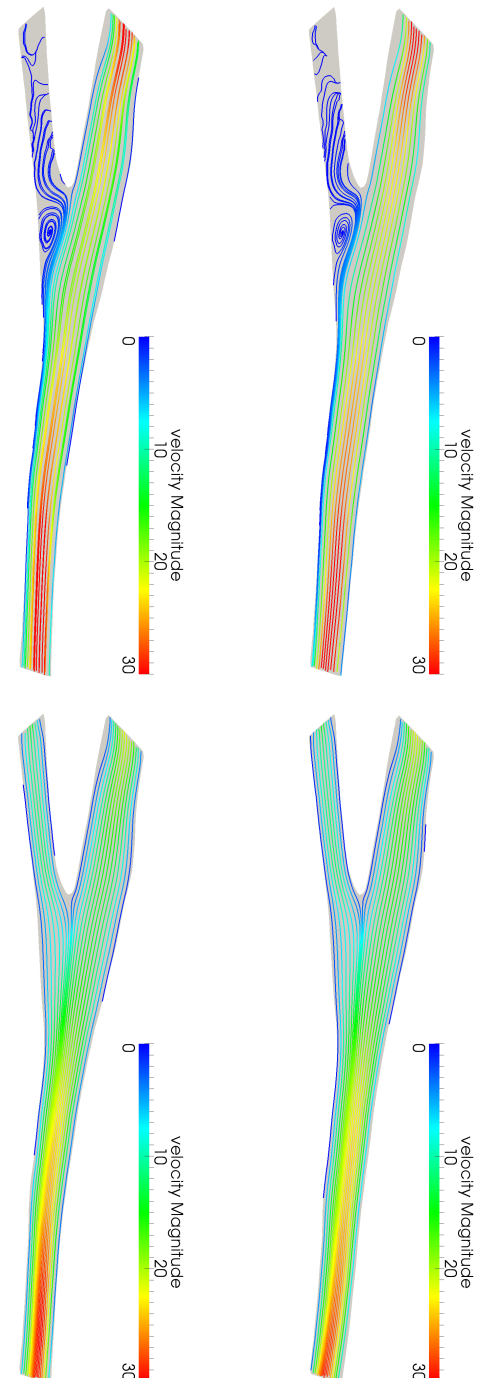

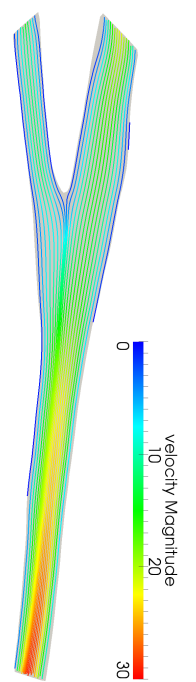

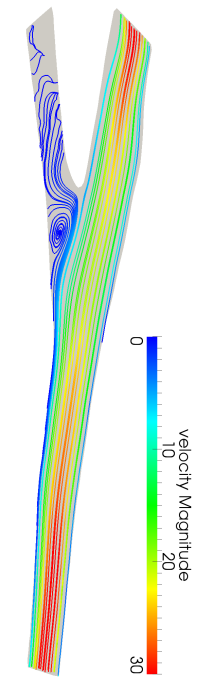

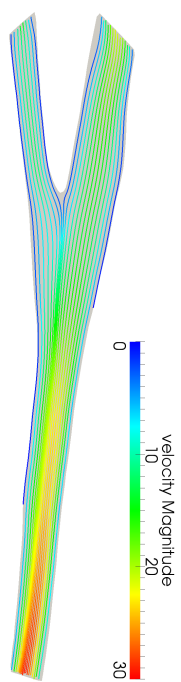

Figure 13. Velocity magnitude and streamlines of the steady incompressible Navier-Stokes (RB simulation); from left to right: initial and optimal configurations obtained with shape optimization of $J_{i}(\boldsymbol{\mu}), i=1,2,3$, using the two extremal values of the residual flow parameter ( $\omega=0$ on top, $\omega=15$ on bottom).

Table II. Results of the shape optimization problem ( $N_{\text {opt }}=16$ cases, $\left.\omega=0,1, \ldots, 15\right)$. Here $\Delta J=\left(J^{(0)}-\hat{J}\right) / J^{(0)}$, and the average is computed over the 16 cases.

\begin{tabular}{lccccc}
\hline & $\Delta J$ (average) & \# optim. iters & \# output evals & \# total output evals & CPU time \\
$J_{1}(\boldsymbol{\mu})$ & 0.5297 & $3 \div 7$ & $28 \div 99$ & 771 & $5.9 \mathrm{~h}$ \\
$J_{2}(\boldsymbol{\mu})$ & 0.6618 & $3 \div 7$ & $28 \div 60$ & 641 & $9.4 \mathrm{~h}$ \\
$J_{3}(\boldsymbol{\mu})$ & 0.7340 & $3 \div 27$ & $28 \div 230$ & 1120 & $11.3 \mathrm{~h}$ \\
\hline
\end{tabular}

A second problem which may be solved within this parametrized reduced framework deals with the possibility to design a bypass graft which is robust with respect to the magnitude of the residual flow across the occluded branch. In other words, we aim at finding the optimal shape of the graft in presence of the worst case scenario in terms of residual flow, to cover the whole range of possible realizations of the uncertain parameter $\omega$, by solving the min-max optimization problem (7).

In particular, we consider only the case of the functionals $J_{1}(\boldsymbol{\mu})$ and $J_{3}(\boldsymbol{\mu})$, since the behavior of the cost functional $J_{2}(\boldsymbol{\mu})$ may be influenced more by the presence of the nonlinear convection than by the vorticity patterns for large values of $\omega$. 
By using the same optimization subroutine we find that the robust configurations correspond, in both cases, to the optimal shapes computed for $\omega=0$ in the previous case (see Fig. 13, columns 2 and 4). In particular, the solution of the robust (shape) optimization problem requires about $\mathcal{O}\left(10^{3}\right)$ input/output evaluations, thus entailing a CPU time which is at least one order of magnitude larger than a (shape) optimization problem (see Table III). This indicates that a design that is robust over the entire range $\omega \in[0,15]$ must be tuned mainly for the case of total occlusion, i.e. $\omega=0$.

Table III. Numerical details about the robust shape optimization problem. Here the number of output evaluations refers to the solution of the minimization problem (for the max $J_{i}(\boldsymbol{\mu})$ functional).

\begin{tabular}{ccccc}
\hline & \# optim. iters & \# output evals & \# total PDE solves & CPU time \\
$J_{1}(\boldsymbol{\mu})$ & 7 & 87 & 1482 & $4.0 \mathrm{~h}$ \\
$J_{3}(\boldsymbol{\mu})$ & 27 & 230 & 3684 & $19.8 \mathrm{~h}$ \\
\hline
\end{tabular}

In the case that total occlusion of the femoral artery is not expected, we can exclude some region near the point of total occlusion and instead consider the range of uncertainty $\omega \in\left[\omega_{\min }, 15\right]$ for some $\omega_{\min }>0$. In the typical case that the amount of occlusion changes over time it is imperative that we should be able to monitor the amount of occlusion over time by simple noninvasive measurements to guarantee that, in fact, $\omega \geq \omega_{\min }$, since otherwise our bypass design will not be robust any more. This leads us naturally to consider the inverse problem of determining the magnitude of the residual flow.

\subsection{Inverse problem of determining residual flow magnitude based on the measured pressure drop}

The results of the shape optimization problems shown in the previous subsection highlight the criticality occurring in presence of a complete blockage of the occluded artery, corresponding to the case $\omega=0$. Indeed, we might be interested in identifying the uncertain residual flow magnitude from some possible, noninvasive measurements of quantities related with the blood flow. In particular, the chance of identifying whether the bypass will be operating under low residual flows or high residual flows might guide in the optimal design of the graft. As in the previous example, our inverse problem is driven by the numerical observation of the mean pressure drop in the bypass:

$$
J_{4}(\boldsymbol{\mu})=\int_{\Gamma_{\text {in }}} p d y-\int_{\Gamma_{\text {out }}} p d y .
$$

The behavior of the mean pressure drop with respect to the parameter $\omega$ in the two robust optimal bypass shapes obtained in the previous analysis is reported in Fig. 14. In both cases a local maximum is observed at $\omega=0$, and the value of the functional decreases as the magnitude of the residual flow increases, but the decrease rate becomes smaller and smaller. This fact yields some interesting consequences in the inverse identification problem. We show the solution of the deterministic inverse problem for two given values of the magnitude of the residual flow $\omega$ :

1. for the robust optimal shape (1), obtained with the functional $J_{1}(\boldsymbol{\mu}), \omega=2.6097$ and $\omega=$ 10.9817, and corresponding observed pressure drops $s^{*}=-440$ and $s^{*}=-490$;

2. for the robust optimal shape (2), obtained with the functional $J_{3}(\boldsymbol{\mu}), \omega=3.6274$ and $\omega=$ 10.0130, and corresponding observed pressure drops $s^{*}=-735$ and $s^{*}=-765$.

The results of the inverse identification problem without regularization are given in Tables IV-V for three particular noise realizations $(\sigma=0.01,0.03,0.05)$, for the robust shapes obtained with the functionals $J_{1}$ and $J_{3}$. Clearly, the less the noise the better the reconstruction.

The estimate becomes increasingly poor for larger amounts of noise, especially for small values of $\omega$, which represent more critical cases not only for the fluid dynamics, but also concerning the decrease rate of the pressure drop. Indeed, also this example shows that the total occlusion represents a critical condition to deal with. 

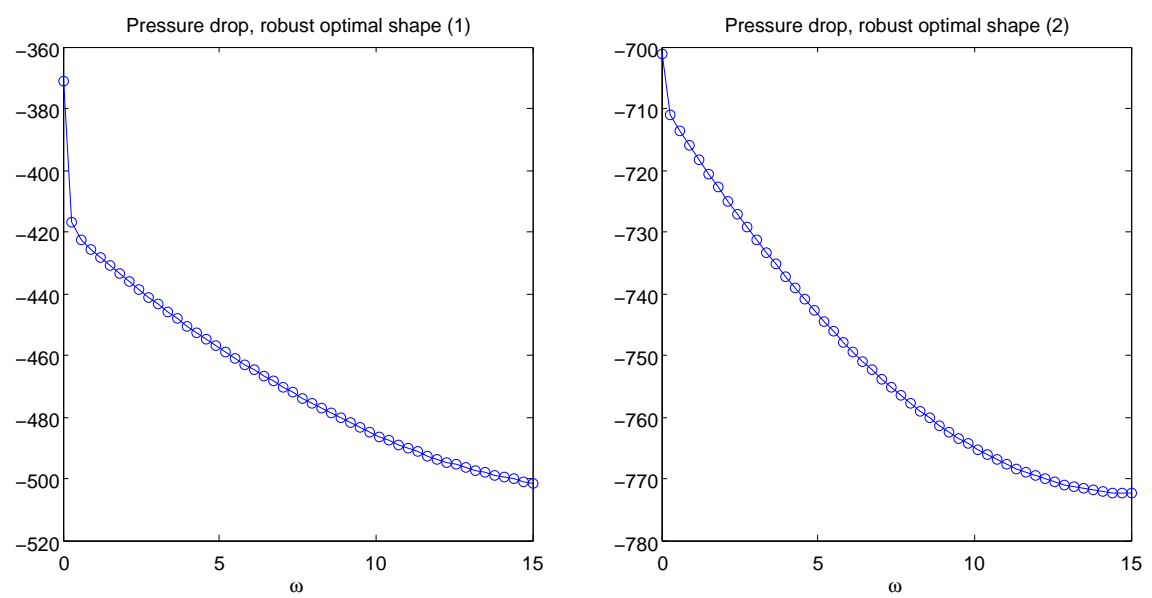

Figure 14. RB evaluation of the mean pressure drop $J_{4}(\hat{\pi}, \omega)$ for different values of $\omega$, for the robust optimal shapes represented by $\hat{\pi}$ and obtained with the cost functionals $J_{1}$ (case 1 , left) and $J_{3}$ (case 2, right).

Table IV. Results of the deterministic inverse problem in two different cases, robust optimal shape (1)

\begin{tabular}{cccccc}
\hline$\omega$ & $\sigma$ & Inverse estimate & Relative error & \# of optim iters & \# of PDE solves \\
\hline 2.6097 & 0.01 & 2.6295 & $0.76 \%$ & 5 & 13 \\
& 0.03 & 3.8004 & $45.63 \%$ & 5 & 13 \\
& 0.05 & 6.3890 & $144.82 \%$ & 4 & 11 \\
\hline \multirow{2}{*}{0.9817} & 0.01 & 11.2151 & $2.13 \%$ & 5 & 13 \\
& 0.03 & 8.2964 & $24.45 \%$ & 4 & 11 \\
& 0.05 & 7.3632 & $32.95 \%$ & 4 & 11 \\
\hline
\end{tabular}

Table V. Results of the deterministic inverse problem in two different cases, robust optimal shape (2)

\begin{tabular}{cccccc}
\hline$\omega$ & $\sigma$ & Inverse estimate & Relative error & \# of optim iters & \# of PDE solves \\
\hline 3.6274 & 0.01 & 3.5594 & $1.87 \%$ & 4 & 11 \\
& 0.03 & 4.1799 & $15.23 \%$ & 6 & 15 \\
& 0.05 & 9.8734 & $172.19 \%$ & 5 & 13 \\
\hline 10.0130 & 0.01 & 9.2899 & $1.83 \%$ & 5 & 13 \\
& 0.03 & 6.6272 & $33.81 \%$ & 4 & 11 \\
& 0.05 & 5.4044 & $46.03 \%$ & 4 & 11 \\
\hline
\end{tabular}

Following the Bayesian approach we provide a probability distribution function for the (uncertain) magnitude of the residual flow $\omega$ encapsulating the noise related to measurements. In particular, if we assume that the prior distribution is normal, $p_{\omega}(\omega) \sim \mathcal{N}\left(\omega_{M}, \tau^{2}\right)$ (we choose a plausible value $\omega_{M}$ for the mean, corresponding to a partial occlusion case, e.g. $\omega_{M}=10$ ), and that the measurements of the pressure drop are $n=250$ independent and normally distributed variables with expectation equal to the true mean pressure drop and variance $\sigma^{2}$, i.e. $p_{s^{*} \mid \omega}\left(s^{*} \mid \omega\right)=$ $p_{\text {noise }}\left(s^{*}-J_{4}(\omega)\right) \sim \mathcal{N}\left(0, \sigma^{2}\right)$, we obtain that also the posterior probability density $p_{\omega \mid s^{*}}\left(\omega \mid s^{*}\right)$ is normally distributed, with mean and variance given by

$$
\mathbb{E}\left[\omega \mid s^{*}\right]=\frac{1 / \tau^{2}}{1 / \tau^{2}+n / \sigma^{2}} \omega_{M}+\frac{n / \sigma^{2}}{1 / \tau^{2}+n / \sigma^{2}} \bar{J}_{4}, \quad \operatorname{Var}\left[\omega \mid s^{*}\right]=\frac{\sigma^{2} / n \tau^{2}}{\tau^{2}+\sigma^{2} / n},
$$

being

$$
\bar{J}_{4}=\frac{1}{n} \sum_{i=1}^{n} J_{4}\left(\omega_{i}\right), \quad i=1, \ldots, n .
$$


Two examples of posterior probability density $p_{\omega \mid s^{*}}\left(\omega \mid s^{*}\right)$, for the two different test cases and their observations $s^{*}$, are shown in Fig. 15. In the situation reported on the left of Fig. 15, the posterior distribution is found to be more distant from the prior distribution, because of the information provided by the measurements. In particular, the posterior p.d.f. is widely/thinly spread and its mean is close/far from the prior mean when the observation noise is small/large, so that, according to (41), the smaller is the observation noise, the more informative is the posterior p.d.f. i.e. the more likely is its mean in identifying the unknown parameter value.

We thus remark that a Bayesian statistic approach can provide a better indication of the identified parameter, including the effect of the measurement noise. Moreover, in the case of a Normal/Normal model, like the one applied in this case, it is simple to evaluate the updating effect provided by the knowledge based on measured quantities.
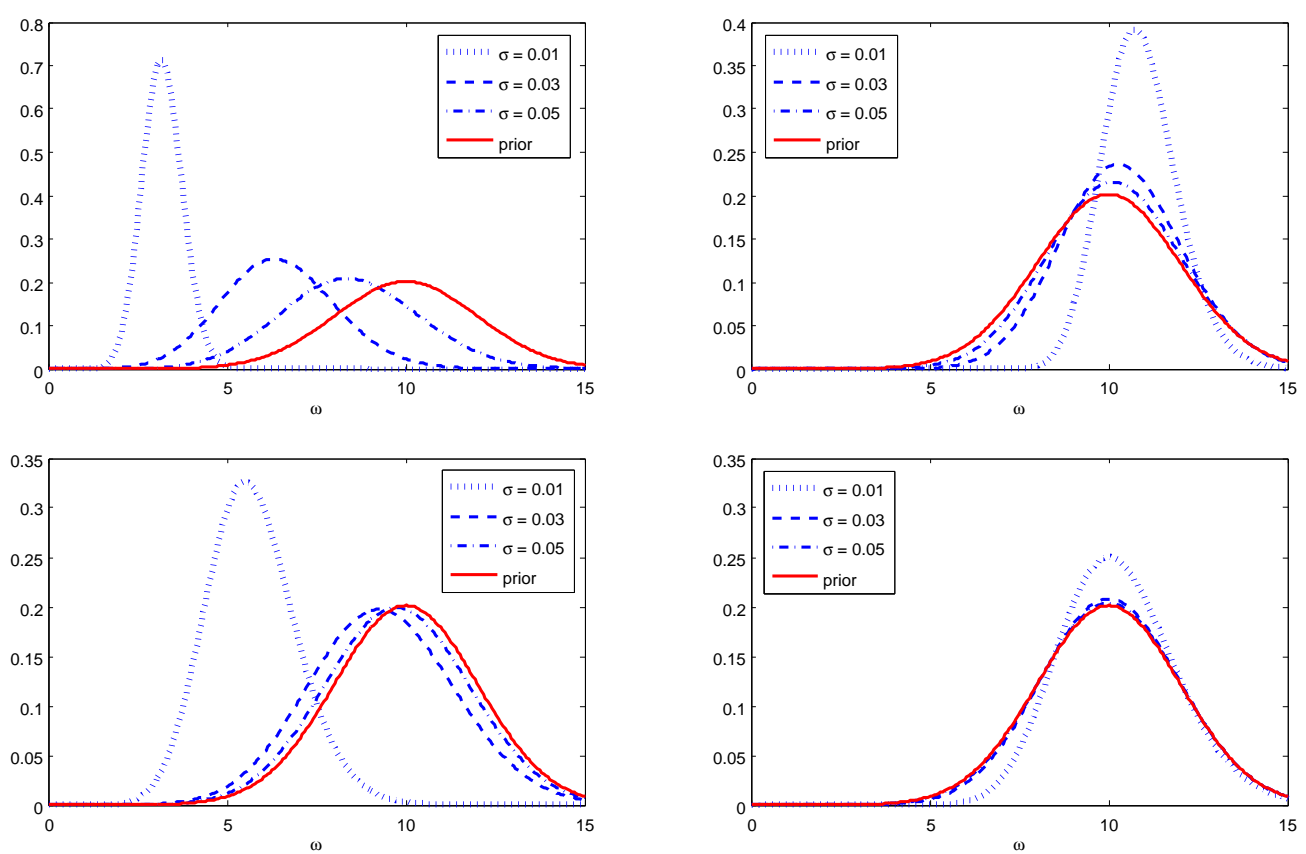

Figure 15. Posterior p.d.f. $p_{\omega \mid s^{*}}\left(\omega \mid s^{*}\right)$ for the robust shapes (1, top) and (3, bottom), in the cases $s^{*}=-440$ (top, left) and $s^{*}=-490$ (top, right), $s^{*}=-735$ (bottom, left) and $s^{*}=-765$ (bottom, right), with three different noise levels: $\sigma^{2}=0.01,0.03,0.05$. In both cases the prior distribution is $p_{\omega}(\omega) \sim \mathcal{N}(10,2)$.

The models we have presented are very simplified, since they do not take into account important features like the pulsatility of the flow and the periodic detachment/separation of the flow layer from the wall. Nevertheless, the exploration of parametrized output as well as the construction of the posterior distribution within the Bayesian framework can be effectively recast in the many-query scenario and treated with the RB method.

The exploration of the parameter space with 250 samples to produce the posterior distributions took around 90 minutes of computational time. The computational speed-up is even stronger for the solution of the robust shape optimization problem: it required up to $\mathcal{O}\left(10^{3}\right)$ PDE solves - even with a very simple geometrical model depending on six input parameters - and took from 4 to 20 hours of computational time, when all the fluid simulations in parametrized configurations were performed by means of the RB method. For the sake of comparison, we estimate that exploring the same parameter space with the full finite element simulation would have taken around 700 hours of computational time - and considerably more if shape deformations had been handled using standard methods and not taking advantage, as done here, of the geometrical reduction afforded by the parametrization of the shape deformation. 


\section{INCLUSION OF THE MODEL REDUCTION ERROR IN THE BAYESIAN ESTIMATOR}

In this section we provide some details on the way a reduced-order model (ROM) - in our case, a RB approximation - affects both the accuracy and the reliability of the solution of an inverse problem. In particular, we wish to incorporate a model for the error engendered by the ROM into the Bayesian estimator - a previous example can be found e.g. in [3]. For the sake of simplicity, we focus on the problem of Sec. 6.3, by considering the case (2), related to the optimal shape obtained with the cost functional $J_{3}(\boldsymbol{\mu})$. We will evaluate the impact of the ROM just on the solution of the inverse problem of recovering the residual flow, encoded by the parameter $\omega$ - the effect of a reduced model on the solution of optimal control and shape optimization problems has already been addressed in previous works (see e.g. [11, 12] for the former case, [44, 42] for the latter).
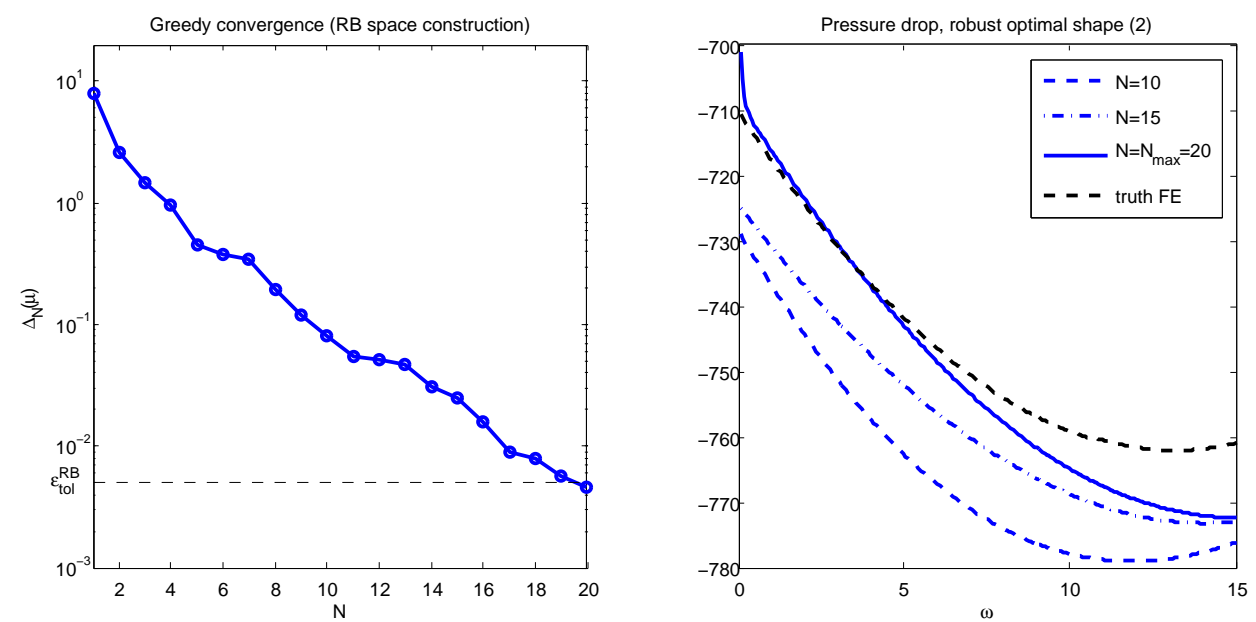

Figure 16. Left: convergence of the greedy algorithm for RB space assembling $\left(N_{\max }=20\right.$ basis are selected with a tolerance $\varepsilon_{t o l}^{R B}=5 \times 10^{-3}$ ). Right: computed mean pressure drop $J_{4}(\boldsymbol{\mu})$ with $N=10,15,20$

basis for the RB solution and the truth FE solution for different values of $\omega$, robust optimal shape (2).

In Fig. 16 (left) we show the convergence of the greedy procedure exploited to assemble the reduced space during the Offline stage; in this particular case, $N_{\max }=20$ basis functions have been selected, by taking advantage, at each step, of the error bound $\Delta_{N}(\boldsymbol{\mu})$ on the flow fields (see Sec. 4). In Fig. 16 (right) we report the mean pressure drop $J_{4}(\boldsymbol{\mu})$ evaluated by using a RB approximation with different reduced model sizes $(N=10,15,20)$ and the truth FE approximation: we can remark that the error between the truth (full-order) FE and the RB outputs becomes smaller as $N$ increases, so that the quality of the reduced-order approximation (measured on flow variables for the sake of flexibility) is thus reflected also on the map $\omega \rightarrow J_{4}(\hat{\pi}, \omega)$ we need to invert $^{\mathrm{h}}$.

We now proceed to evaluate the effect of the reduced-order approximation on the solution of the inverse problem in the Bayesian framework, by incorporating the approximation error in the Bayes' formula. We start by replacing the truth approximation in the additive noise model (14):

$$
s^{*}=s_{h}(\boldsymbol{\mu})+\varepsilon,
$$

which is motivated by $s_{h}(\boldsymbol{\mu})$ being sufficiently close to $s(\boldsymbol{\mu})$ - this is the reason why we call it "truth" approximation ${ }^{\mathrm{i}}$.

\footnotetext{
${ }^{h}$ Since the basis functions cluster in the interval $\omega<5$, the quality of the output approximation is better in this region; we also remark that the computed basis correspond to different shape configurations - in fact, they depend also on $P_{\pi}=6$ geometrical parameters - whereas the outputs of Fig. 16 are evaluated on a fixed shape, which is obtained through the minimization of the $J_{3}(\boldsymbol{\mu})$ functional. This explains the apparently imprecise approximation for $\omega>10$.

${ }^{i}$ As explained in Sect. 4, the RB field solution $U_{N}(\boldsymbol{\mu})$ and RB output $s_{N}(\boldsymbol{\mu})$ shall approximate the "truth" finite element field solution $U_{h}(\boldsymbol{\mu})$ and and output $s_{h}(\boldsymbol{\mu})$. The reduced basis error is, in fact, evaluated with respect to the "truth" finite element solution.
} 
Then, since we rely on a ROM approximation $s_{N}(\boldsymbol{\mu})$ of the output, we can rewite (42) as

$$
s^{*}=s_{N}(\boldsymbol{\mu})+\eta(\boldsymbol{\mu})=s_{N}(\boldsymbol{\mu})+\left(s_{h}(\boldsymbol{\mu})-s_{N}(\boldsymbol{\mu})\right)+\varepsilon,
$$

where $\eta(\boldsymbol{\mu})=\left(s_{h}(\boldsymbol{\mu})-s_{N}(\boldsymbol{\mu})\right)+\varepsilon$ is the total error model including both the ROM approximation error and the measurement noise $\varepsilon$. Consequently, the likelihood is:

$$
p_{s^{*}} \mid \boldsymbol{\mu}\left(s^{*} \mid \boldsymbol{\mu}\right)=p\left(s_{N}(\boldsymbol{\mu})+\eta(\boldsymbol{\mu}) \mid \boldsymbol{\mu}\right) .
$$

Although in principle the approximation error $s_{h}(\boldsymbol{\mu})-s_{N}(\boldsymbol{\mu})$ is deterministic, in practice its evaluation is out of reach since it would require the solution of the full-order model. Thus, we treat this epistemic (or systematic) uncertainty ${ }^{\mathrm{j}}$ as a random variable, as was done in the so-called approximation error model framework presented in [3].

In this way, by assuming that the measurement noise (affecting the observation process) and the approximation error (affecting the ROM output) are independent and normally distributed with zero mean and variances $\sigma^{2}$ and $\sigma_{r}^{2}$, respectively, we have that

$$
\eta \sim \mathcal{N}\left(0, \sigma^{2}+\sigma_{r}^{2}\right)
$$

and the likelihood function is

$$
p_{s^{*} \mid \boldsymbol{\mu}}\left(s^{*} \mid \boldsymbol{\mu}\right) \propto \exp \left(-\frac{1}{2\left(\sigma^{2}+\sigma_{r}^{2}\right)}\left\|s^{*}-s_{N}(\boldsymbol{\mu})\right\|^{2}\right) .
$$

The relationship (13) enables then to compute the posterior distribution $p_{\boldsymbol{\mu} \mid s^{*}}\left(\boldsymbol{\mu} \mid \mathbf{s}^{*}\right)$, as was done in (15). In this way, it is possible to compare the relative impact of the two error sources on the posterior distributions we obtain through the inversion process, as shown in the example below.

First, in Fig. 17 we compare the posterior distributions obtained with different ROMs (for $N=10,15,20)$ and with the full-order FE approximation, by considering two different level of noise error, $\sigma^{2}=0.01$ and $\sigma^{2}=0.05$, without taking into account any approximation error $\left(\sigma_{r}^{2}=0\right)$.

As we can see, if the noise variance $\sigma^{2}$ is quite large, the posterior distribution is not informative, since the contribution provided by the prior distribution is more relevant - independently of the quality of the reduced model, i.e. of the number $N \in\left[1, N_{\max }\right]$ of basis we select (see Fig. 17, right). Instead, if the noise variance $\sigma^{2}$ is rather small, the ROM quality greatly affects the posterior distribution, and then the estimate (see Fig. 17, left): the larger the dimension $N$ of the RB approximation is, the closer the posterior distribution becomes to the one obtained using the truth FE solution. Nevertheless, for small values of $N$, the estimate might result very biased because of the approximation error.

Thus, the ROM error has only an effect if the measurement noise is very small, but without incorporating the ROM error in the Bayes' formula, the posterior estimate is not sufficiently robust. Instead, if we include also the approximation error in the Bayes' formula, by choosing in (44)

$$
\sigma_{r}^{2}=\max _{\mu}\left|\Delta_{N}(\mu)\right|
$$

and fixing the same noise variances $\sigma^{2}$ and basis numbers $N$ as in Fig. 17, we obtain the posterior distributions represented in Fig. 18. As we can see, in the case of small noise variance (see Fig. 18, left), the posterior distribution obtained with a poor ROM is less biased and closer to the prior distribution if we take into account also the approximation error $\sigma_{r}$ in the likelihood function. In this way, the knowledge provided by the error bounds on flow fields can be exploited to quantify the approximation error also in the inverse problem. The situation is even more clear in Fig. 19, where a smaller noise variance $\sigma^{2}=0.005$ has been chosen: in the case of a ROM approximation

\footnotetext{
${ }^{j}$ See for instance [16] for several remarks about the distinction between epistemic (or systematic) uncertainty and aleatoric (or statistical) uncertainty.
} 

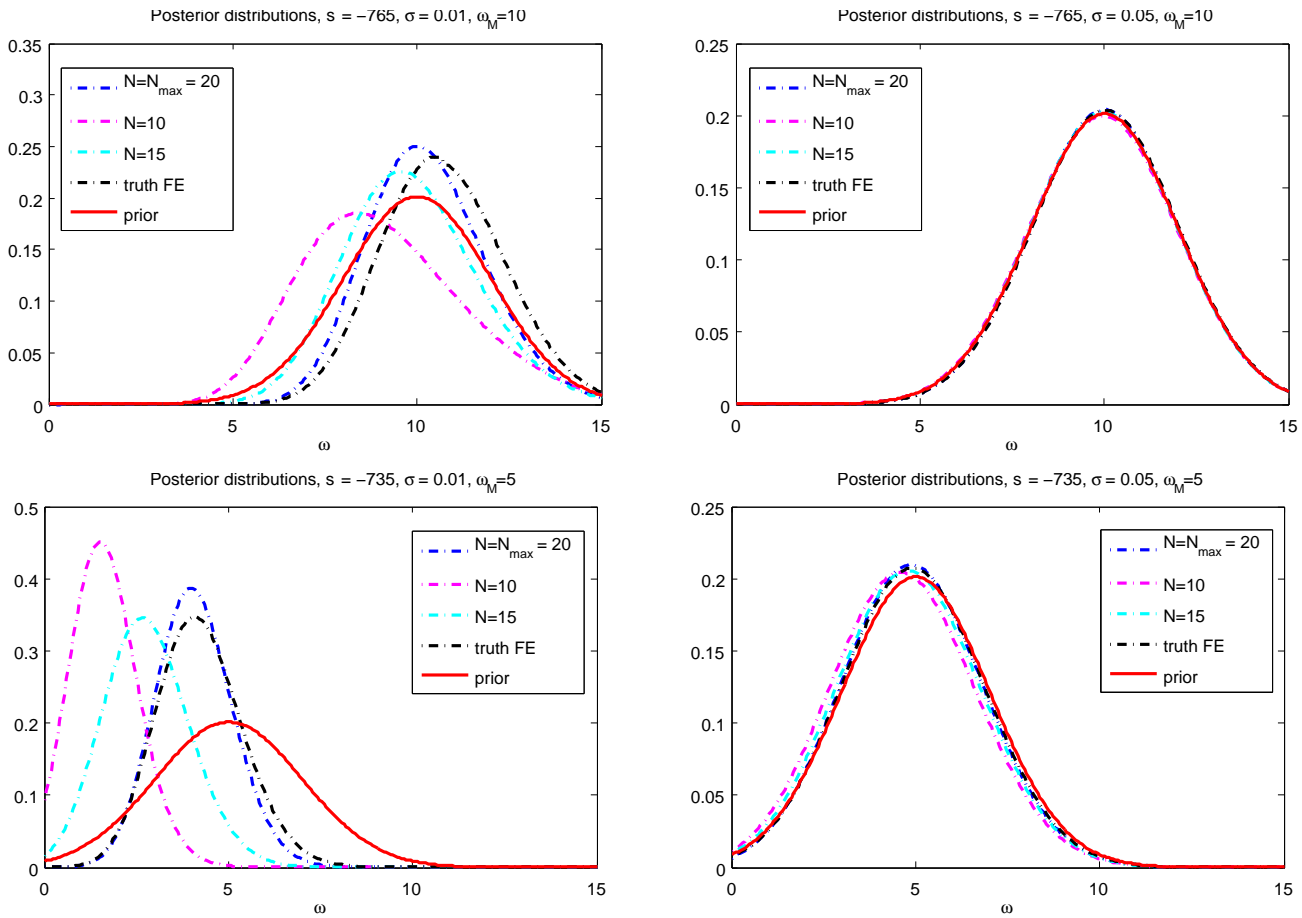

Figure 17. Robust shape (2): posterior p.d.f. $p_{\omega \mid s^{*}}\left(\omega \mid s^{*}\right)$ obtained with $\mathrm{RB}(N=10,15,20)$ and $\mathrm{FE}$ approximations of the flow solution, for the cases $s^{*}=-735$ (top) and $s^{*}=-765$ (bottom) with two different noise levels: $\sigma^{2}=0.01$ (left) and $\sigma^{2}=0.05$ (right). The prior distributions are $p_{\omega}(\omega) \sim \mathcal{N}\left(\omega_{M}, 2\right)$, being $\omega_{M}=10$ (top) and $\omega_{M}=5$ (bottom).
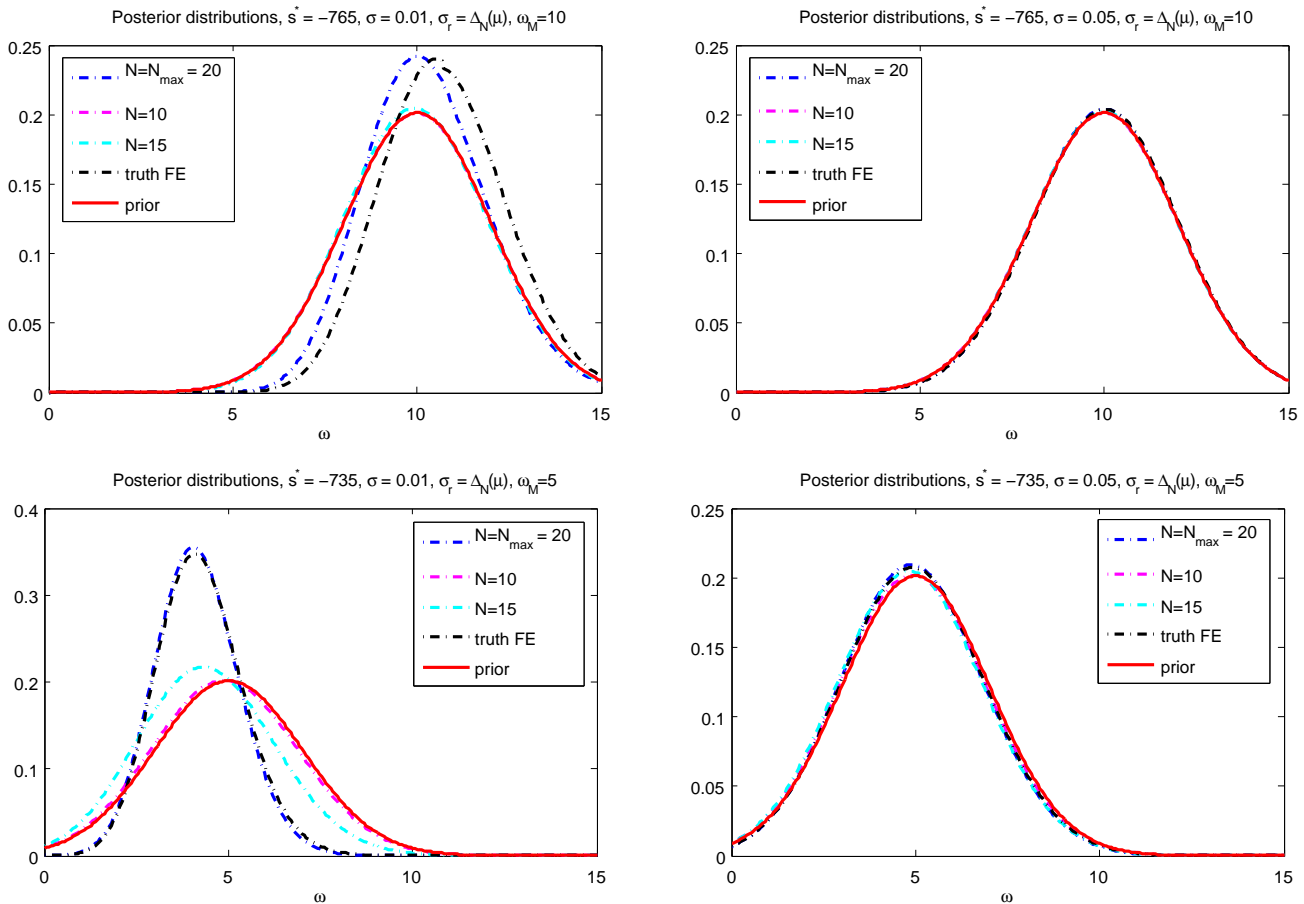

Figure 18. Robust shape (2): posterior p.d.f. $p_{\omega \mid s^{*}}\left(\omega \mid s^{*}\right)$ obtained with $\mathrm{RB}(N=10,15,20)$ and FE evaluations of the flow solution, for the cases $s^{*}=-735$ (top) and $s^{*}=-765$ (bottom), with two different noise errors: $\sigma_{n}^{2}=0.01$ (left) and $\sigma_{n}^{2}=0.05$ (right), and reduction error $\sigma_{r}=\max _{\mu} \Delta_{N}(\mu)$. The prior distributions are $p_{\omega}(\omega) \sim \mathcal{N}\left(\omega_{M}, 2\right)$, being $\omega_{M}=10$ (top) and $\omega_{M}=5$ (bottom). 
which is too poor, the inclusion of the approximation error in the model allows to obtain a posterior distribution which is even more thinly spread in the case of a larger ROM dimension, and even closer to the prior distribution in the case of a smaller ROM dimension, thus confirming the opportunity to introduce an error term due to the ROM approximation. Instead, if the noise $\sigma^{2}$ is quite large compared to the approximation error $\sigma_{r}^{2}$, we obtain non-informative posterior distributions, like in the previous situation (see Fig. 18, right).

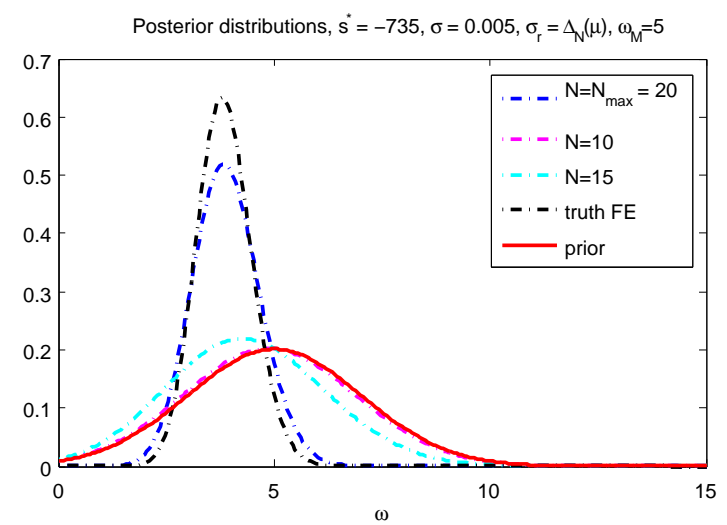

Figure 19. Robust shape (2): posterior p.d.f. $p_{\omega \mid s^{*}}\left(\omega \mid s^{*}\right)$ obtained with $\mathrm{RB}(N=10,15,20)$ and FE evaluations of the flow solution, for the case $s^{*}=-735$ with noise error $\sigma_{n}^{2}=0.005$ and reduction error $\sigma_{r}^{2}=\max _{\boldsymbol{\mu}}\left|\Delta_{N}(\boldsymbol{\mu})\right|$. The prior distribution is $p_{\omega}(\omega) \sim \mathcal{N}(5,2)$.

We point out that more rigorous estimates could be provided by error bounds evaluated directly on the output at hand (in this case, the mean pressure drop) - however, this task would require the solution (and the RB approximation) of a further, dual problem, which is output-dependent, thus violating the flexibility purpose of our procedure. Furthermore, this model could be extended by considering a $\boldsymbol{\mu}$-dependent expression for the variance $\sigma_{r}^{2}$, that could be evaluated by looking directly at the error estimate $\Delta_{N}(\boldsymbol{\mu})$ : however, the identification of the covariance structure of the $\mathrm{ROM}$ error process is not in general an easy task, and is outside of the goals of this paper.

\section{CONCLUSIONS AND REMARKS}

We presented a computational and geometrical model reduction framework for treating some haemodynamic inverse problems considered as test cases. The computational reduction framework is guaranteed by using the Reduced Basis method, while the geometrical one is ensured by resorting to Free-Form Deformation techniques used to parametrize the domain.

The framework was successfully applied to two inverse problem in haemodynamics. In the first example we formulated a steady fluid-structure interaction problem in a portion of a stenosed artery; by solving the inverse problem of haemodynamics we predicted downstream shear rates linked to stenosis growth given a noninvasive pressure drop measurement. This inversion could be performed even in the likely case that the pressure measurements are too noisy to accurately identify the vessel wall material parameters themselves. In the second example we considered the optimal shape design of a femoro-popliteal bypass graft anastomoses under unknown residual flow magnitude. The robust design of the anastomosis depended mostly on the least allowed residual flow magnitude. The critical case of total occlusion in the femoral artery could be either identified or ruled out based on similar noninvasive pressure drop measurements. Thus it was possible to ascertain whether or not the bypass was operating under the design conditions for which robustness was guaranteed. 
We also showed how the reduced-order approximation of the direct problem affects the solution of an inverse problem in the Bayesian framework: we can argue that this framework, exploiting the available information on the approximation error coming from the reduced-order model, enables to get a more reliable and robust solution of the inverse problem.

The models we have presented are very simplified, since they do not take into account important features like the pulsatility of the flow and the periodic detachment/separation of the flow layer from the wall. Nevertheless, the reduced models presented allow to characterize (almost) in real-time blood flows in 2D geometries, by capturing several features related e.g. to vessel shape, fluid behavior and also structural parameters. In particular, the coupling between geometrical reduction and computational reduction proves to be necessary in order to tackle optimal shape design and more in general for inverse problems related with shape variation (even for these sample problems). The adoption of 2D geometries, as well as the assumption of steady flows, have been introduced in order to reduce the computational costs. Nevertheless, several features inherited also by 3D flows in simplified geometries can be highlighted already in corresponding 2D configurations, e.g. in the case of an idealized optimal control formulation for graft design [35].

In view of applying the proposed methodology to problems of practical interest in haemodynamics, we need to extend the current framework to $(i)$ more realistic 3D configurations and (ii) unsteady flows, already taken into consideration in several problems solved by traditional discretization schemes (also in presence of uncertainties). These developments are the focus of future research work, parallel to the extension of the RB methodology (and its certification). Within the framework analyzed in this paper, the extension of the proposed geometrical reduction techniques is straightforward, by developing automatic procedures for constructing shape parametrizations in order to manage more complex shape configurations. This task can be accomplished using modern data assimilation and image reconstruction techniques (see e.g. [61] for preliminary results).

\section{APPENDIX A - FLOW MODELLING: PARAMETRIZED NAVIER-STOKES EQUATIONS}

In this Appendix we briefly recall the reduced basis approximation of the incompressible steady Navier-Stokes equations [52, 43], used to model blood flows in the examples discussed throughout the paper. We start from the weak formulation in the original domain, we introduce an equivalent formulation in the reference domain, and we conclude by showing how to compute the reduced basis approximation, highlighting the computational stages based on the Offline/Online decomposition.

The weak formulation of Navier-Stokes equations in the original domain $\Omega_{o}(\boldsymbol{\pi})$ reads as follows: find $U_{o}(\boldsymbol{\mu})=\left(\boldsymbol{u}_{o}(\boldsymbol{\mu}), p_{o}(\boldsymbol{\mu})\right) \in X\left(\Omega_{o}(\boldsymbol{\pi})\right)$ s.t.

$$
\begin{aligned}
& \int_{\Omega_{0}(\boldsymbol{\pi})}[\nu(\boldsymbol{\omega}) \nabla \boldsymbol{u}: \nabla \boldsymbol{v}+(\boldsymbol{u} \cdot \nabla) \boldsymbol{u} \cdot \boldsymbol{v}-p \nabla \cdot \boldsymbol{v}] d \Omega \\
& +\int_{\Omega_{0}(\boldsymbol{\pi})}\left[\left(\boldsymbol{g}_{o}(\boldsymbol{\omega}) \cdot \nabla\right) \boldsymbol{u} \cdot \boldsymbol{v}+(\boldsymbol{u} \cdot \nabla) \boldsymbol{g}_{o}(\boldsymbol{\omega}) \cdot \boldsymbol{v}\right] d \Omega=\int_{\Omega_{\mathrm{o}}(\boldsymbol{\pi})} \boldsymbol{f}(\boldsymbol{\omega}) \cdot \boldsymbol{v} d \Omega \\
& -\int_{\Omega_{0}(\boldsymbol{\pi})} \nu(\boldsymbol{\omega}) \nabla \boldsymbol{g}_{o}(\boldsymbol{\omega}): \nabla \boldsymbol{v} d \Omega-\int_{\Omega_{0}(\boldsymbol{\pi})}\left(\boldsymbol{g}_{o}(\boldsymbol{\omega}) \cdot \nabla\right) \boldsymbol{g}_{o}(\boldsymbol{\omega}) \cdot \boldsymbol{v} d \Omega, \quad \forall \boldsymbol{v} \in V\left(\Omega_{o}(\boldsymbol{\pi})\right) \\
& -\int_{\Omega_{0}(\boldsymbol{\pi})} q \nabla \cdot \boldsymbol{u} d \Omega=\int_{\Omega_{\mathrm{o}}(\boldsymbol{\pi})} q \nabla \cdot \boldsymbol{g}_{o}(\boldsymbol{\omega}) d \Omega \\
& \forall q \in Q\left(\Omega_{o}(\boldsymbol{\pi})\right)
\end{aligned}
$$

where the dependence on $\boldsymbol{\mu}=(\boldsymbol{\pi}, \boldsymbol{\omega})$ has been omitted in the velocity and pressure fields for the sake of simplicity. Here $X\left(\Omega_{o}(\boldsymbol{\pi})\right)=\left(H_{0, \Gamma_{D}}^{1}\left(\Omega_{o}(\boldsymbol{\pi})\right)\right)^{2} \times L^{2}\left(\Omega_{o}(\boldsymbol{\pi})\right)$, while $\boldsymbol{g}_{o} \in H^{1}\left(\Omega_{o}(\boldsymbol{\pi})\right)$ is a lifting of the inhomogeneous Dirichlet boundary conditions, and $H_{0, \Gamma_{D}}^{1}\left(\Omega_{o}\right):=\left\{v \in H^{1}\left(\Omega_{o}\right)\right.$ : $\left.\left.v\right|_{\Gamma_{D}}=0\right\}$. We assume that the Neumann condition are only of homogeneous type. 
By mapping this problem back to the reference domain $\Omega$, we obtain the following parametrized weak formulation: find $U(\boldsymbol{\mu})=(\boldsymbol{u}(\boldsymbol{\mu}), p(\boldsymbol{\mu})) \in X$ s.t.

$$
\mathcal{A}(U(\boldsymbol{\mu}), W ; \boldsymbol{\mu})+\mathcal{C}(U(\boldsymbol{\mu}), U(\boldsymbol{\mu}), W ; \boldsymbol{\mu})=\mathcal{F}(W ; \boldsymbol{\mu}), \quad \forall W \in X,
$$

where $X=V \times Q=\left(H_{0, \Gamma_{D}}^{1}(\Omega)\right)^{2} \times L^{2}(\Omega)$ and

$$
\begin{gathered}
\mathcal{A}(U, W ; \boldsymbol{\mu})=a(\boldsymbol{u}, \boldsymbol{w} ; \boldsymbol{\mu})+d(\boldsymbol{u}, \boldsymbol{w} ; \boldsymbol{\mu})+b(p, \boldsymbol{w} ; \boldsymbol{\mu})+b(q, \boldsymbol{u} ; \boldsymbol{\mu}), \\
\mathcal{C}(U, V, W ; \boldsymbol{\mu})=c(\boldsymbol{u}, \boldsymbol{v}, \boldsymbol{w} ; \boldsymbol{\mu}), \\
\mathcal{F}(W ; \boldsymbol{\mu})=\left\langle F^{s}(\boldsymbol{\mu}), \boldsymbol{w}\right\rangle+\left\langle F^{l}(\boldsymbol{\mu}), \boldsymbol{w}\right\rangle+\left\langle G^{l}(\boldsymbol{\mu}), q\right\rangle .
\end{gathered}
$$

In this case,

$$
\begin{array}{cr}
a(\boldsymbol{v}, \boldsymbol{w} ; \boldsymbol{\mu})=\int_{\Omega} \frac{\partial \boldsymbol{v}}{\partial x_{i}} \nu_{i j}(\mathbf{x}, \boldsymbol{\mu}) \frac{\partial \boldsymbol{w}}{\partial x_{j}} d \Omega, & \boldsymbol{\nu}(\boldsymbol{x}, \boldsymbol{\mu})=\nu(\boldsymbol{\omega}) \boldsymbol{J}_{T}^{-1} \boldsymbol{J}_{T}^{-T}\left|\boldsymbol{J}_{T}\right|, \\
b(p, \boldsymbol{w} ; \boldsymbol{\mu})=-\int_{\Omega} p \chi_{i j}(\mathbf{x}, \boldsymbol{\mu}) \frac{\partial w_{j}}{\partial x_{i}} d \Omega, & \boldsymbol{\chi}(\boldsymbol{x}, \boldsymbol{\mu})=\boldsymbol{J}_{T}^{-1}\left|\boldsymbol{J}_{T}\right|, \\
c(\boldsymbol{u}, \boldsymbol{v}, \boldsymbol{w} ; \boldsymbol{\mu})=\int_{\Omega} u_{i} \chi_{i j}(\boldsymbol{x}, \boldsymbol{\mu}) \frac{\partial v_{k}}{\partial x_{j}} w_{k} d \Omega, &
\end{array}
$$

being $\boldsymbol{J}_{T}=\boldsymbol{J}_{T}(\boldsymbol{x}, \boldsymbol{\mu})$ the Jacobian of $T(\boldsymbol{x}, \boldsymbol{\mu})$ and $\left|\boldsymbol{J}_{T}\right|$ its determinant. In the same way, we have

$$
\left\langle F^{s}(\boldsymbol{\mu}), \boldsymbol{w}\right\rangle=\int_{\Omega} \mathbf{f} \cdot \boldsymbol{w}\left|\boldsymbol{J}_{T}\right| d \Omega,
$$

while $d(\boldsymbol{v}, \boldsymbol{w} ; \boldsymbol{\mu})=c(\boldsymbol{g}, \boldsymbol{v}, \boldsymbol{w} ; \boldsymbol{\mu})+c(\boldsymbol{v}, \boldsymbol{g}, \boldsymbol{w} ; \boldsymbol{\mu}),\left\langle F^{l}(\boldsymbol{\mu}), \boldsymbol{w}\right\rangle=-a(\boldsymbol{g}, \boldsymbol{w} ; \boldsymbol{\mu})-c(\boldsymbol{g}, \boldsymbol{g}, \boldsymbol{w} ; \boldsymbol{\mu})$ and $\left\langle G^{l}(\boldsymbol{\mu}), q\right\rangle=-b(q, \boldsymbol{g} ; \boldsymbol{\mu})$ are terms due to non-homogeneous Dirichlet boundary conditions, while $\boldsymbol{g} \in\left(H^{1}(\Omega)\right)^{2}$ is a lifting function related to the reference domain $\Omega$.

The finite element approximation of the problem (46) is obtained as a Galerkin projection onto the space $X_{h}=V_{h} \times Q_{h}$, being $V_{h} \subset V, Q_{h} \subset Q$ two sequences of FE approximation spaces of global dimension $\mathcal{N}_{h}=\mathcal{N}_{X}+\mathcal{N}_{Q}$ : find $U_{h}(\boldsymbol{\mu})=\left(\boldsymbol{u}_{h}(\boldsymbol{\mu}), p_{h}(\boldsymbol{\mu})\right) \in X_{h}$ s.t.

$$
\mathcal{A}\left(U_{h}(\boldsymbol{\mu}), W ; \boldsymbol{\mu}\right)+\mathcal{C}\left(U_{h}(\boldsymbol{\mu}), U_{h}(\boldsymbol{\mu}), W ; \boldsymbol{\mu}\right)=\mathcal{F}(W ; \boldsymbol{\mu}), \quad \forall W \in X_{h} .
$$

The equivalent matrix formulation of problem (53) thus reads as follows:

$$
\left[\begin{array}{cc}
\mathbb{A}_{h}(\boldsymbol{\mu})+\mathbb{C}_{h}\left(\boldsymbol{u}_{h}(\boldsymbol{\mu}) ; \boldsymbol{\mu}\right) & \mathbb{B}_{h}^{T}(\boldsymbol{\mu}) \\
\mathbb{B}_{h}(\boldsymbol{\mu}) & \mathbb{O}
\end{array}\right]\left[\begin{array}{l}
\boldsymbol{u}_{h}(\boldsymbol{\mu}) \\
\mathbf{p}_{h}(\boldsymbol{\mu})
\end{array}\right]=\left[\begin{array}{c}
\mathbb{F}_{h}(\boldsymbol{\mu}) \\
\mathbb{G}_{h}(\boldsymbol{\mu})
\end{array}\right]
$$

being, for $1 \leq i, j \leq \mathcal{N}_{X}, 1 \leq k \leq \mathcal{N}_{Q}$,

$$
\begin{gathered}
\left(\mathbb{A}_{h}(\boldsymbol{\mu})\right)_{i j}=a\left(\boldsymbol{\xi}_{j}^{h}, \boldsymbol{\xi}_{i}^{h} ; \boldsymbol{\mu}\right)+c\left(\boldsymbol{g}^{h}, \boldsymbol{\xi}_{j}^{h}, \boldsymbol{\xi}_{i}^{h} ; \boldsymbol{\mu}\right)+c\left(\boldsymbol{\xi}_{j}^{h}, \boldsymbol{g}^{h}, \boldsymbol{\xi}_{i}^{h} ; \boldsymbol{\mu}\right) \\
\left(\mathbb{B}_{h}(\boldsymbol{\mu})\right)_{k i}=b\left(\varphi_{k}^{h}, \boldsymbol{\xi}_{i}^{h} ; \boldsymbol{\mu}\right), \quad\left(\mathbb{C}_{h}(\boldsymbol{w} ; \boldsymbol{\mu})\right)_{i j}=c\left(\sum_{k=1}^{\mathcal{N}_{X}} w_{k}^{h} \boldsymbol{\xi}_{k}^{h}, \boldsymbol{\xi}_{j}^{h}, \boldsymbol{\xi}_{i}^{h} ; \boldsymbol{\mu}\right), \\
\left(\mathbb{F}_{h}(\boldsymbol{\mu})\right)_{i}=\left\langle F^{s}(\boldsymbol{\mu}), \boldsymbol{\xi}_{i}^{h}\right\rangle+\left\langle F^{l}(\boldsymbol{\mu}), \boldsymbol{\xi}_{i}^{h}\right\rangle, \quad\left(\mathbb{G}_{h}(\boldsymbol{\mu})\right)_{k}=\left\langle G^{l}(\boldsymbol{\mu}), \varphi_{k}^{h}\right\rangle,
\end{gathered}
$$

where $\left\{\boldsymbol{\xi}_{i}^{h}\right\}_{i=1}^{\mathcal{N}_{X}}$ and $\left\{\varphi_{i}^{h}\right\}_{i=1}^{\mathcal{N}_{Q}}$ denote two bases of the spaces $V_{h}$ and $Q_{h}$, respectively. Moreover, $\boldsymbol{u}_{h}(\boldsymbol{\mu})=\left(u_{i}^{h}(\boldsymbol{\mu})\right)_{i=1}^{\mathcal{N}_{X}}$ and $\mathbf{p}_{h}(\boldsymbol{\mu})=\left(p_{k}^{h}\right)_{k=1}^{\mathcal{N}_{Q}}$ are the vectors corresponding to (the nodal values of) velocity and pressure, and $\boldsymbol{g}_{h}$ is the FE interpolant of non-homogeneous Dirichlet data.

The nonlinear system (54) is solved by means of a fixed-point iteration, using as initial guess the Stokes solution $\left[\boldsymbol{u}_{h}^{(0)}(\boldsymbol{\mu}), \mathbf{p}_{h}^{(0)}(\boldsymbol{\mu})\right]$ obtained by dropping the convective terms; for $k>0$, the 
following linearized (Oseen) problem has to be solved:

$$
\left[\begin{array}{cc}
\mathbb{A}_{h}(\boldsymbol{\mu})+\mathbb{C}_{h}\left(\boldsymbol{u}_{h}^{(k-1)}(\boldsymbol{\mu}) ; \boldsymbol{\mu}\right) & \mathbb{B}_{h}^{T}(\boldsymbol{\mu}) \\
\mathbb{B}_{h}(\boldsymbol{\mu}) & \mathbb{O}
\end{array}\right]\left[\begin{array}{c}
\boldsymbol{u}_{h}^{(k)}(\boldsymbol{\mu}) \\
\mathbf{p}_{h}^{(k)}(\boldsymbol{\mu})
\end{array}\right]=\left[\begin{array}{c}
\mathbb{F}_{h}(\boldsymbol{\mu}) \\
\mathbb{G}_{h}(\boldsymbol{\mu})
\end{array}\right]
$$

until a given stopping criterion (say, $\left\|\boldsymbol{u}_{h}^{(k)}(\boldsymbol{\mu})-\boldsymbol{u}_{h}^{(k-1)}(\boldsymbol{\mu})\right\|_{2} \leq \varepsilon_{\text {tol }}^{N S}$ ) is fulfilled.

The reduced basis method enables to compute an approximation of $U_{h}(\boldsymbol{\mu})$ by using the global approximation spaces

$$
\begin{gathered}
Q_{N}=\operatorname{span}\left\{\phi_{n}=p_{h}\left(\boldsymbol{\mu}^{n}\right), n=1, \ldots, N\right\}, \\
V_{N}=\operatorname{span}\left\{\sigma_{n}:=\boldsymbol{u}_{h}\left(\boldsymbol{\mu}^{n}\right), T_{p}^{\mu} \phi_{n}, n=1, \ldots, N\right\} ;
\end{gathered}
$$

$T_{p}^{\mu}: Q_{N} \rightarrow V_{N}$ is such that $\left(T_{p}^{\mu} q, \boldsymbol{w}\right)_{X}=b(q, \boldsymbol{w} ; \boldsymbol{\mu}) \quad \forall \boldsymbol{w} \in V_{N}$ and is called inner supremizer operator, The enrichment of the velocity space by means of the supremizer solutions enables to fulfill an inf-sup stability condition also on the RB spaces [62, 58], as usually done in the FE context in order to get a stable couple of velocity/pressure spaces.

The RB approximation of the Navier-Stokes problem (46) is thus obtained through a Galerkin projection onto $X_{N}=V_{N} \times Q_{N}$ : find $U_{N}(\boldsymbol{\mu})=\left(\boldsymbol{u}_{N}(\boldsymbol{\mu}), p_{N}(\boldsymbol{\mu})\right) \in X_{N}$ s.t.

$$
\mathcal{A}\left(U_{N}(\boldsymbol{\mu}), W ; \boldsymbol{\mu}\right)+\mathcal{C}\left(U_{N}(\boldsymbol{\mu}), U_{N}(\boldsymbol{\mu}), W ; \boldsymbol{\mu}\right)=\mathcal{F}(W ; \boldsymbol{\mu}), \quad \forall W \in X_{N} .
$$

Following the same procedure used for the FE case, problem (56) can be written under matrix form:

$$
\left[\begin{array}{cc}
\mathbb{A}_{N}(\boldsymbol{\mu})+\mathbb{C}_{N}\left(\boldsymbol{u}_{N}(\boldsymbol{\mu}) ; \boldsymbol{\mu}\right) & \mathbb{B}_{N}^{T}(\boldsymbol{\mu}) \\
\mathbb{B}_{N}(\boldsymbol{\mu}) & \mathbb{O}
\end{array}\right]\left[\begin{array}{l}
\boldsymbol{u}_{N}(\boldsymbol{\mu}) \\
\mathbf{p}_{N}(\boldsymbol{\mu})
\end{array}\right]=\left[\begin{array}{c}
\mathbb{F}_{N}(\boldsymbol{\mu}) \\
\mathbb{G}_{N}(\boldsymbol{\mu})
\end{array}\right]
$$

being, for $1 \leq m, n \leq 2 N, 1 \leq r \leq N$,

$$
\begin{gathered}
\left(\mathbb{A}_{N}(\boldsymbol{\mu})\right)_{m n}=a\left(\boldsymbol{\xi}_{n}^{N}, \boldsymbol{\xi}_{m}^{N} ; \boldsymbol{\mu}\right)+c\left(\boldsymbol{g}^{h}, \boldsymbol{\xi}_{n}^{N}, \boldsymbol{\xi}_{m}^{N} ; \boldsymbol{\mu}\right)+c\left(\boldsymbol{\xi}_{n}^{N}, \boldsymbol{g}^{h}, \boldsymbol{\xi}_{m}^{N} ; \boldsymbol{\mu}\right) \\
\left(\mathbb{B}_{N}(\boldsymbol{\mu})\right)_{r m}=b\left(\varphi_{r}^{N}, \boldsymbol{\xi}_{m}^{N} ; \boldsymbol{\mu}\right), \quad\left(\mathbb{C}_{N}\left(\boldsymbol{w}_{N} ; \boldsymbol{\mu}\right)\right)_{m n}=\sum_{s=1}^{2 N} w_{N s} c\left(\boldsymbol{\xi}_{s}^{N}, \boldsymbol{\xi}_{n}^{N}, \boldsymbol{\xi}_{m}^{N} ; \boldsymbol{\mu}\right), \\
\left(\mathbb{F}_{N}(\boldsymbol{\mu})\right)_{m}=\left\langle F^{s}(\boldsymbol{\mu}), \boldsymbol{\xi}_{m}^{N}\right\rangle+\left\langle F^{s}(\boldsymbol{\mu}), \boldsymbol{\xi}_{m}^{N}\right\rangle, \quad\left(\mathbb{G}_{N}(\boldsymbol{\mu})\right)_{r}=\left\langle G^{l}(\boldsymbol{\mu}), \varphi_{r}^{N}\right\rangle
\end{gathered}
$$

where $\left\{\boldsymbol{\xi}_{n}^{N}\right\}_{i=1}^{2 N}$ and $\left\{\varphi_{r}^{N}\right\}_{r=1}^{N}$ denote two bases of the spaces $V_{N}$ and $Q_{N}$, respectively. Moreover, $\boldsymbol{u}_{N}(\boldsymbol{\mu})=\left(u_{n}^{N}(\boldsymbol{\mu})\right)_{i=1}^{2 N}$ and $\mathbf{p}_{N}(\boldsymbol{\mu})=\left(p_{s}^{N}\right)_{s=1}^{N}$ are the vectors corresponding to the basis components of velocity and pressure. As for the FE case, (57) is solved by means of a fixed-point iteration, using as initial guess the RB Stokes solution $\left[\boldsymbol{u}_{N}^{(0)}(\boldsymbol{\mu}), \mathbf{p}_{N}^{(0)}(\boldsymbol{\mu})\right]$. We recall that the RB structures are obtained from the corresponding FE structures by means of a pre/post multiplication by the spaces matrices (25).

In the end, since shape parametrizations based on FFD mappings give rise to nonaffine tensors (at least for the viscous terms) an additional computational stage is required during the Offline stage in order to recover the affinity assumption - crucial for the Offline/Online decomposition itself, and thus for the computational speedup. To do this, we rely on an empirical interpolation of the parametrized tensors appearing in the bilinear/trilinear forms; a detailed description of this procedure for the Stokes case can be found in [44]; the extension to the Navier-Stokes case is straightforward. 


\section{ACKNOWLEDGEMENTS}

We acknowledge, in the framework of the Progetto Roberto Rocca (MIT-Politecnico di Milano), Prof. A.T. Patera (MIT) for his insights, as well as all the people who have contributed with him to the rbMIT package used for RB computations presented in this work. We thank Dr. J. Bonnemain (EPFL/Centre Hospitalier Universitaire Vaudois) for his valuable feedbacks and suggestions. This work has been supported in part by the Swiss National Science Foundation (Project 200021-122136) and by the ERC-Mathcard Project (Project ERC-2008-AdG 227058).

\section{REFERENCES}

1. V. Agoshkov, A. Quarteroni, and G. Rozza. A mathematical approach in the design of arterial bypass using unsteady Stokes equations. J. Sci. Comput., 28(2-3):139-165, 2006.

2. E.I. Amoiralis and I.K. Nikolos. Freeform deformation versus B-spline representation in inverse airfoil design. $J$. Comput. Inf. Sci. Engrg., 8, 2008.

3. S.R. Arridge, J.P. Kaipio, V. Kolehmainen, M. Schweiger, E. Somersalo, T. Tarvainen, and M. Vauhkonen. Approximation errors and model reduction with an application in optical diffusion tomography. Inverse Problems, 22(1):175-195, 2006.

4. S. Balocco, O. Basset, G. Courbebaisse, E. Boni, A.F. Frangi, P. Tortoli, and C. Cachard. Estimation of the viscoelastic properties of vessel walls using a computational model and Doppler ultrasound. Phys. Med. Biol., 55(12):3557-3575, 2010.

5. S. Balocco, O. Basset, G. Courbebaisse, E. Boni, P. Tortoli, and C. Cachard. Noninvasive Young's modulus evaluation of tissues surrounding pulsatile vessels using ultrasound Doppler measurement. IEEE Trans. Ultrason. Ferroelectr. Freq. Control., 54(6):1265-1271, 2007.

6. M. Barrault, Y. Maday, N.C. Nguyen, and A.T. Patera. An 'empirical interpolation' method: application to efficient reduced-basis discretization of partial differential equations. C. R. Math. Acad. Sci. Paris, 339(9):667-672, 2004.

7. C. Bertoglio, P. Moireau, and J.-F. Gerbeau. Sequential parameter estimation for fluid-structure problems. Application to hemodynamics. Int. J. Num. Meth. Biomed. Engn., 28(4):434-455, 2012.

8. S. Boyaval, C. Le Bris, T. Lelièvre, Y. Maday, N.C. Nguyen, and A.T. Patera. Reduced basis techniques for stochastic problems. Arch. Comput. Methods Engrg., 17(4):1-20, 2010.

9. T. F. Coleman and Y. Li. An interior trust region approach for nonlinear minimization subject to bounds. SIAM J. Optim., 6:418-445, 1996.

10. A.R. Conn, N.I.M. Gould, and Ph.L. Toint. Trust Region Methods. MOS-SIAM Ser. Optim. Society for Industrial and Applied Mathematics, Philadephia, PA, 2000.

11. L. Dedè. Reduced basis method and a posteriori error estimation for parametrized linear-quadratic optimal control problems. SIAM J. Sci. Comput., 32(2):997-1019, 2010.

12. L. Dedè. Reduced basis method and error estimation for parametrized optimal control problems with control constraints. J. Sci. Comput., 50(2):287-305, 2012.

13. M. D'Elia, L. Mirabella, T. Passerini, M. Perego, M. Piccinelli, C. Vergara, and A. Veneziani. Applications of variational data assimilation in computational hemodynamics. In: D. Ambrosi, A. Quarteroni, G. Rozza (Eds.) Modeling of Physiological Flows, Springer-Verlag Italia, 2011.

14. S. Deparis. Reduced basis error bound computation of parameter-dependent Navier-Stokes equations by the natural norm approach. SIAM J. Num. Anal., 46(4):2039-2067, 2008.

15. S. Deparis and G. Rozza. Reduced basis method for multi-parameter-dependent steady Navier-Stokes equations: Applications to natural convection in a cavity. J. Comp. Phys., 228(12):4359-4378, 2009.

16. A. Der Kiureghian and O. Ditlevsen. Aleatory or epistemic? Does it matter? Structural Safety, 31(2):105-112, 2009.

17. C.R. Ethier, D.A. Steinman, X. Zhang, S.R. Karpik, and M. Ojha. Flow waveform effects on end-to-side anastomotic flow patterns. J. Biomech., 31(7):609-617, 1998.

18. R. Fletcher. Modified Marquardt subroutine for non-linear least squares. Technical report, Atomic Energy Research Establishment, Harwell (England), 1971.

19. L. Formaggia, J.-F. Gerbeau, F. Nobile, and F. Quarteroni. On the coupling of 3D and 1D Navier-Stokes equations for flow problems in compliant vessels. Comput. Methods Appl. Mech. Engrg., 191(6-7):561-582, 2001.

20. M. Frangos, Y. Marzouk, K. Willcox, and B. van Bloemen Waanders. Surrogate and reduced-order modeling: A comparison of approaches for large-scale statistical inverse problems. Large-Scale Inverse Probl. Quantification Uncertainty, pages 123-149, 2011.

21. J. Gain and D. Bechmann. A survey of spatial deformation from a user-centered perspective. ACM Trans. Graph., 27(4):107:1-107:21, 2008

22. D. Galbally, K. Fidkowski, K. Willcox, and O. Ghattas. Nonlinear model reduction for uncertainty quantification in large-scale inverse problems. Int. J. Numer. Methods Engrg, 81(12):1581-1608, 2010.

23. M.D. Gunzburger. Perspectives in Flow Control and Optimization. Advances in Design and Control. Society for Industrial and Applied Mathematics, Philadephia, PA, 2003.

24. M. Hintermüller, K. Kunisch, Y. Spasov, and S. Volkwein. Dynamical systems-based optimal control of incompressible fluids. Int. J. Numer. Meth. Fluids, 46(4):345-359, 2004.

25. J.D. Humphrey. Continuum biomechanics of soft biological tissues. Proc. R. Soc. A, 459(2029):3-46, 2003.

26. D.B.P. Huynh, D.J. Knezevic, and A.T. Patera. Certified reduced basis model characterization: a frequentistic uncertainty framework. Comput. Methods Appl. Mech. Engrg., 201-204:13-24, 2012. 
27. D.B.P. Huynh, N.C. Nguyen, A.T. Patera, and G. Rozza. Rapid reliable solution of the parametrized partial differential equations of continuum mechanics and transport, 2008-2011. Website: http://augustine.mit.edu.

28. D.B.P Huynh, G. Rozza, S. Sen, and A.T. Patera. A successive constraint linear optimization method for lower bounds of parametric coercivity and inf-sup stability costants. C. R. Acad. Sci. Paris. Sér. I Math., 345:473-478, 2007.

29. J. Kaipio and E. Somersalo. Statistical and computational inverse problems. Springer Science+Business Media, Inc., 2005.

30. H. Kasumba and K. Kunisch. Shape design optimization for viscous flows in a channel with a bump and an obstacle. In Methods and Models in Automation and Robotics (MMAR), 2010 15th International Conference on, pages 284-289. IEEE.

31. R.S. Keynton, M.M. Evancho, R.L. Sims, N.V. Rodway, A. Gobin, and S.E. Rittgers. Intimal hyperplasia and wall shear in arterial bypass graft distal anastomoses: an in vivo model study. J. Biomech. Engr., 123:464, 2001.

32. D.J. Knezevic, N.C. Nguyen, and A.T. Patera. Reduced basis approximation and a posteriori error estimation for the parametrized unsteady Boussinesq equations. Math. Mod. and Meth. in Appl. Sc., 21(7):1415-1442, 2011.

33. D.N. Ku, D.P. Giddens, C.K. Zarins, and S. Glagov. Pulsatile flow and atherosclerosis in the human carotid bifurcation. positive correlation between plaque location and low oscillating shear stress. Arterioscler. Thromb. Vasc. Biol., 5(3):293-302, 1985.

34. K. Kunisch and B. Vexler. Optimal vortex reduction for instationary flows based on translation invariant cost functionals. SIAM J. Control Optim, 46(4):1368-1397, 2007.

35. T. Lassila, A. Manzoni, A. Quarteroni, and G. Rozza. Boundary control and shape optimization for the robust design of bypass anastomoses under uncertainty. ESAIM Math. Modelling Numer. Anal., 2013. in press.

36. T. Lassila, A. Quarteroni, and G. Rozza. A reduced basis model with parametric coupling for fluid-structure interaction problem. SIAM J. Sci. Comput., 34(2):A1187-A1213, 2012

37. T. Lassila and G. Rozza. Model reduction of steady fluid-structure interaction problems with free-form deformations and reduced basis methods. Proc. 10th Finnish Mech. Days, Jyväskylä, Finland, December 2009, pages 454-465, 2009.

38. T. Lassila and G. Rozza. Parametric free-form shape design with PDE models and reduced basis method. Comput. Methods Appl. Mech. Engrg., 199(23-24):1583-1592, 2010.

39. C. Lieberman, K. Willcox, and O. Ghattas. Parameter and state model reduction for large-scale statistical inverse problems. SIAM J. Sci. Comput., 32(5):2523-2542, 2010.

40. A. Lipponen, A. Seppänen, and J.P. Kaipio. Reduced-order estimation of nonstationary flows with electrical impedance tomography. Inverse Probl., 26, 2010.

41. F. Loth, S.A. Jones, D.P. Giddens, H.S. Bassiouny, S. Glagov, and C.K. Zarins. Measurements of velocity and wall shear stress inside a PTFE vascular graft model under steady flow conditions. J. Biomech. Engr., 119:187, 1997.

42. A. Manzoni. Reduced models for optimal control, shape optimization and inverse problems in haemodynamics. $\mathrm{PhD}$ thesis, École Polytechnique Fédérale de Lausanne, 2012.

43. A. Manzoni, A. Quarteroni, and G. Rozza. Model reduction techniques for fast blood flow simulation in parametrized geometries. Int. J. Numer. Methods Biomed. Engng., 28((6-7)):604-625, 2012.

44. A. Manzoni, A. Quarteroni, and G. Rozza. Shape optimization of cardiovascular geometries by reduced basis methods and free-form deformation techniques. Int. J. Numer. Methods Fluids, 70(5):646-670, 2012.

45. K. Mosegaard and A. Tarantola. Probabilistic approach to inverse problems. In P. Jennings W. Lee, H. Kanamori and C. Kisslinger, editors, International Handbook of Earthquake and Engineering Seismology, volume 81, Part A, pages 237-265. Academic Press, 2002.

46. N.C. Nguyen, G. Rozza, D.B.P. Huynh, and A.T. Patera. Reduced basis approximation and a posteriori error estimation for parametrized parabolic PDEs: Application to real-time Bayesian parameter estimation. In L. Biegler, G. Biros, O. Ghattas, M. Heinkenschloss, D. Keyes, B. Mallick, Y. Marzouk, L. Tenorio, B. van Bloemen Waanders, and K. Willcox (Eds.), Large-Scale Inverse Problems and Quantification of Uncertainty,, John Wiley \& Sons, Ltd, $U K, 2009$.

47. J. Nocedal and S.J. Wright. Numerical optimization. Springer Verlag, 1999.

48. J.J. Oliver and D.J. Webb. Noninvasive assessment of arterial stiffness and risk of atherosclerotic events. Arteriolscl. Throm. Vas., 23(4):554-566, 2003.

49. A.T. Patera and G. Rozza. Reduced Basis Approximation and A Posteriori Error Estimation for Parametrized Partial Differential Equation. Version 1.0, Copyright MIT 2006, to appear in (tentative rubric) MIT Pappalardo Graduate Monographs in Mechanical Engineering, 2009.

50. M. Perego, A. Veneziani, and C. Vergara. Variational approach for estimating the compliance of the cardiovascular tissue: an inverse fluid-structure interaction problem. SIAM J. Sci. Comp., 33(3):1181-1211, 2011.

51. A. Quarteroni and G. Rozza. Optimal control and shape optimization of aorto-coronaric bypass anastomoses. Math. Models Meth. Appl. Sci., 13(12):1801-1823, 2003.

52. A. Quarteroni and G. Rozza. Numerical solution of parametrized Navier-Stokes equations by reduced basis methods. Numer. Methods Partial Differential Equations, 23(4):923-948, 2007.

53. A. Quarteroni, G. Rozza, and A. Manzoni. Certified reduced basis approximation for parametrized partial differential equations in industrial applications. J. Math. Ind., 1(3), 2011.

54. A. Quarteroni, M. Tuveri, and A. Veneziani. Computational vascular fluid dynamics: problems, models and methods. Comp. Vis. Sci., 2:163-197, 2000.

55. C.M. Quick, D.S. Berger, R.H. Stewart, G.A. Laine, C.J. Hartley, and A. Noordergraaf. Resolving the hemodynamic inverse problem. IEEE Trans. Biomed. Eng, 53(3):361-368, 2006.

56. J.O. Ramsay and B.W. Silverman. Applied functional data analysis: methods and case studies. Springer Verlag, 2002.

57. A.M. Robertson, A. Sequeira, and M.V. Kameneva. Hemorheology. Hemodynamical Flows, pages 63-120, 2008.

58. G. Rozza, D.B.P. Huynh, and A. Manzoni. Reduced basis approximation and a posteriori error estimation for Stokes flows in parametrized geometries: roles of the inf-sup stability constants. Numer. Math. (in press), 2013. 
Online version: http://infoscience.epfl.ch/record/182350? ln=en.

59. G. Rozza, D.B.P. Huynh, and A.T. Patera. Reduced basis approximation and a posteriori error estimation for affinely parametrized elliptic coercive partial differential equations. Arch. Comput. Methods Engrg., 15:229-275, 2008.

60. G. Rozza, T. Lassila, and A. Manzoni. Reduced basis approximation for shape optimization in thermal flows with a parametrized polynomial geometric map. In Spectral and High Order Methods for Partial Differential Equations. Selected papers from the ICOSAHOM '09 conference, June 22-26, Trondheim, Norway, pages 307-315. Springer, Series: Lecture Notes in Computational Science and Engineering, vol. 76, J.S. Hesthaven, E.M. Rønquist (Eds.), 2011.

61. G. Rozza, A. Manzoni, and F. Negri. Reduction strategies for pde-constrained optimization problems in haemodynamics. In J. Eberhardsteiner et.al., editor, Proceedings of the European Congress on Computational Methods in Applied Sciences and Engineering (ECCOMAS 2012), Vienna, Austria, September 10-14, 2012, 2012.

62. G. Rozza and K. Veroy. On the stability of reduced basis methods for Stokes equations in parametrized domains. Comput. Methods Appl. Mech. Engrg., 196(7):1244-1260, 2007.

63. J. Sainte-Marie, D. Chapelle, R. Cimrman, and M. Sorine. Modeling and estimation of the cardiac electromechanical activity. Comput. Struct., 84:1743-1759, 2006.

64. S. Sankaran and A.L. Marsden. The impact of uncertainty on shape optimization of idealized bypass graft models in unsteady flow. Phys. Fluids, 22:121902, 2010.

65. T.W. Sederberg and S.R. Parry. Free-form deformation of solid geometric models. Comput. Graph., 20(4), 1986.

66. M. Sermesant, P. Moireau, O. Camara, Sainte-Marie J., R. Andriantsimiavona, R. Cimrman, D.L.G. Hill, D. Chapelle, and R. Razavi. Cardiac function estimation from MRI using a heart model and data assimilation: Advances and difficulties. Medical Image Anal., 10:642-656, 2006.

67. A.M. Stuart. Inverse problems: a Bayesian perspective. Acta Numer., 19(1):451-559, 2010.

68. N.M. van Popele, D.E. Grobbee, M.L. Bots, R. Asmar, J. Topouchian, R.S. Reneman, A.P.G. Hoeks, D.A.M. van der Kuip, A. Hofman, and J.C.M. Witteman. Association between arterial stiffness and atherosclerosis: the Rotterdam study. Stroke, 32(2):454-460, 2001.

69. K. Veroy and A.T. Patera. Certified real-time solution of the parametrized steady incompressible Navier-Stokes equations: rigorous reduced-basis a posteriori error bounds. Int. J. Numer. Meth. Fluids, 47(8-9):773-788, 2005.

70. V.Y. Wang, HI Lam, D.B. Ennis, B.R. Cowan, A.A. Young, and M.P. Nash. Modelling passive diastolic mechanics with quantitative MRI of cardiac structure and function. Medical Image Anal., 13(5):773-784, 2009.

71. T. Weber, J. Auer, M.F. O’Rourke, E. Kvas, E. Lassnig, R. Berent, and B. Eber. Arterial stiffness, wave reflections, and the risk of coronary artery disease. Circulation, 109(2):184-189, 2004.

72. K. Zaw, G.R. Liu, B. Deng, and K.B.C. Tan. Rapid identification of elastic modulus of the interface tissue on dental implants surfaces using reduced-basis method and a neural network. J. Biomech., 42(5):634-641, 2009. 